\title{
Aeroelastic Tailoring of the NASA Common Research Model via Novel Material and Structural Configurations
}

\author{
Christine V. Jutte \\ Craig Technologies, Inc., Cape Canaveral, FL 32920 \\ Bret K. Stanford, Carol D. Wieseman, and James B. Moore \\ NASA Langley Research Center, Hampton, VA 23681
}

\begin{abstract}
This work explores the use of tow steered composite laminates, functionally graded metals (FGM), thickness distributions, and curvilinear rib/spar/stringer topologies for aeroelastic tailoring. Parameterized models of the Common Research Model (CRM) wing box have been developed for passive aeroelastic tailoring trade studies. Metrics of interest include the wing weight, the onset of dynamic flutter, and the static aeroelastic stresses. Compared to a baseline structure, the lowest aggregate static wing stresses could be obtained with tow steered skins (47\% improvement), and many of these designs could reduce weight as well (up to $14 \%$ ). For these structures, the trade-off between flutter speed and weight is generally strong, although one case showed both a $100 \%$ flutter improvement and a $3.5 \%$ weight reduction. Material grading showed no benefit in the skins, but moderate flutter speed improvements (with no weight or stress increase) could be obtained by grading the spars $(4.8 \%)$ or ribs $(3.2 \%)$, where the best flutter results were obtained by grading both thickness and material. For the topology work, large weight reductions were obtained by removing an inner spar, and performance was maintained by shifting stringers forward and/or using curvilinear ribs: $5.6 \%$ weight reduction, a $13.9 \%$ improvement in flutter speed, but a 3.0\% increase in stress levels. Flutter resistance was also maintained using straightrotated ribs although the design had a $4.2 \%$ lower flutter speed than the curved ribs of similar weight and stress levels were higher. These results will guide the development of a future design optimization scheme established to exploit and combine the individual attributes of these technologies.
\end{abstract}

\section{Introduction}

The Fixed Wing project of NASA's Fundamental Aeronautics program has been actively developing manufacturing techniques, new materials, and structural design tools to address a suite of technical challenges facing current and future subsonic transport aircraft. A primary challenge of the Fixed Wing project is to reduce fuel burn in transport aircraft. Targeted design advancements include wing structural weight reduction and increased wing aspect ratio to decrease lift-induced drag. High aspect ratio wings operating at minimum weight are typically highly flexible structures prone to aeroelastic instabilities; this paper studies the application of several novel material and structural technologies for aeroelastic tailoring to mitigate these issues.

Aeroelastic tailoring can be defined as "the embodiment of directional stiffness into an aircraft structural design to control aeroelastic deformation, static or dynamic, in such a fashion as to affect the aerodynamic and structural performance of that aircraft in a beneficial way," [1]. More simply, aeroelastic tailoring has also been defined as "passive aeroelastic control" [2]. Traditionally, tailoring has been achieved with composite shell structures via bend-twist coupling causing either wash-in (tip leading edge up) or wash-out (tip leading edge down). Tailoring the primary stiffness direction of a laminated wing structure can have a profound effect on the aero-structural attributes, many of which can only be improved at the expense of others [1]. Traditional aeroelastic tailoring has focused on stiffness-based methods (via composite materials), though the mass distribution (via inertial loads) can have a large effect upon the aeroelastic behavior as well. This has been historically less of a factor during initial wing design and more to mitigate harmful unforeseen dynamics found later in the design process. Inclusion of these metrics at an early stage may be expected to provide superior structures compared to traditional design methods. Certain tailoring parameterizations can alter the stiffness distribution but not the inertial distribution, some vice-versa, and others impact both simultaneously. 
The general concept of aeroelastic tailoring is not new [1]; and a large number of papers have been published on the subject, some of which are referenced in this section. It is found, however, that many of these papers utilize a simplified wing structure: a beam or a flat plate. This is with good reason: a simplified treatment allows trends and trade-offs to be clearly seen and understood. In this work, however, a fully-populated wing box structure (within the Common Research Model (CRM) wing [3]) is used to take advantage of more complex configurations and tailoring schemes that modify the internal structural arrangement and material properties. It is also found in the literature that relatively few papers consider multiple aeroelastic metrics, and those that do, use typically conflicting metrics, so this will be emphasized here as well.

The current work is focused on tailoring schemes that have been recently enabled by advances in materials or manufacturing methods. Three are considered, as shown in Figure 1: fiber tow steered composites, functionally graded materials (FGM), and curvilinear ribs/spars/stringers. The first technology is enabled by an automated fiber tow placement machine (where composite fibers are applied along curvilinear paths within the plane of a laminate), the latter two by metal additive manufacturing processes like electron beam freeform fabrication $\left(\mathrm{EBF}^{3}\right)$ [4]. None of these methods have been extensively used for aeroelastic tailoring and/or aircraft structural design (largely due to their novelty), but some existing works can be found in the literature, as will be noted below.

Many papers that consider the design of tow steered aerospace structures are confined to single panels subject to buckling [5], frequency [6], or strength [7] considerations. Panels with cutouts are of particular interest [7], as tailored load paths can potentially alleviate the stress concentrations around the cutout (window, bay door, etc.). Similar cut-out based considerations are applicable to wing design as well, and these strategies may also prove useful at the junctions of spars and skins (and the resulting stress concentration), for example. Expanding from single panels to entire wing structures, few papers exist in the literature. Haddadpour and Zamani [8] modeled a subsonic cantilevered wing as a thin-walled beam and altered the steering paths of the composite skins to maximize the aeroelastic flutter speed. Stodieck et al. [9] modeled the wing as a plate-like structure and demonstrated the relationship between the tow steering pattern and various aeroelastic metrics of interest: flexural axis, divergence, flutter, and gust loads.
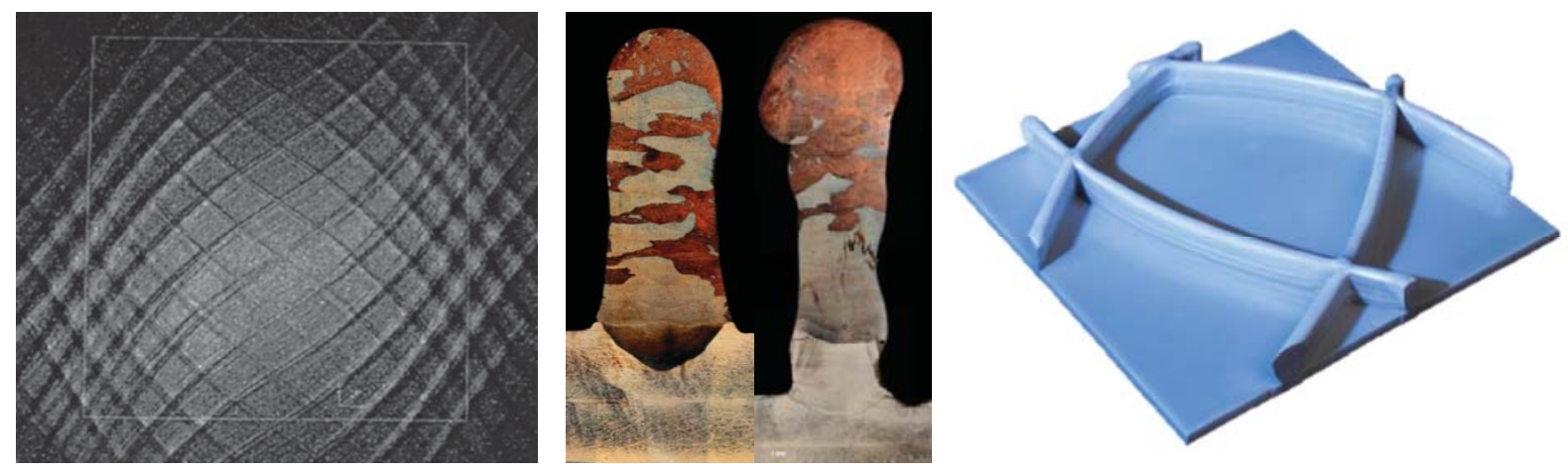

Figure 1. Structures manufactured with the tailoring technologies considered in this paper: tow steered composites (left), functionally graded metallic (center), and curvilinear wing reinforcement (right). Images courtesy $\mathrm{C}$. Wu and $\mathrm{K}$. Taminger, NASA LaRC.

Similar to tow steering, much of the literature pertaining to FGM in an aerospace setting is confined to the panel level. In particular, most of the existing work details the use of FGM for elastic panels subject to supersonic flows (and thus aerodynamic heating): see Ref. [10] for a review of this work. Material grading is beneficial to hightemperature applications as it eliminates discrete changes in the coefficient of thermal expansion, which can lead to stress concentrations at material boundaries. Aerothermoelastic panel flutter boundaries have been shown to benefit as well. Despite the potential of FGMs to improve the structural performance of subsonic wings [11], very little work has been done. Librescu and Maalawi [12] used material grading to optimize the material distribution of a cantilevered subsonic wing (maximization of torsional divergence under a mass constraint). Linear, parabolic, and piecewise material grading distributions along the wing span were all considered. Though this paper used fiber volume fractions of composite materials (rather than metallic grading, which is the emphasis of the current work), it is notable as one of very few papers that considers subsonic aeroelastic tailoring via material grading.

Curvilinear structural reinforcement of wing structures, like the previous two examples, has been readily demonstrated at the panel level, with the majority of the work conducted by Kapania and coworkers at Virginia Tech. Curved stiffeners on metallic panels (right side of Figure 1, for example) have been shown to improve the 
performance of a minimum-mass panel under buckling, crippling, and strength constraints [13], [14]. Locatelli et al. [15] expanded the concept to the wing box level, using full-depth curvilinear ribs and spars. Curvilinear structures were again shown to effectively minimize wing weight under a variety of constraints, though aeroelastic physics and metrics were not considered. Finally, though Refs. [16], [17], and [18] do not utilize a curvilinear parameterization, all demonstrated a benefit in structural weight/performance due to a deviation from the traditional orthogonal grid of metallic ribs and spars within a wing box.

For the present work, an all-metallic single-material baseline wing box structure was developed for the CRM, consisting of wing skins, an orthogonal grid of primary rib and spar structures, and secondary stringer and ribstiffened structures. An aeroelastic framework was developed using a suite of MATLAB, PATRAN, and NASTRAN modules to automate computation of the static aeroelastic strength and the dynamic aeroelastic flutter boundary of a given wing structure. These concepts are described in Section II and III. Sections IV, V, and VI explore the use of tow steered composites, material/thickness grading, and curvilinear ribs/spars/stringers for the aeroelastic tailoring of the CRM. This is largely done using parameter sweeps or limited design space samplings. The structural weight, aeroelastic strength, and flutter behavior of each design permutation is continually compared to the performance of the aforementioned baseline wing box structure. Sections IV, V, and VI are summaries of detailed design studies which will be individually published in the near future as NASA Technical Memorandums. Section VII discusses the results of the studies, and VIII provides general recommendations for future work.

\section{Baseline Description}

The transport aircraft wing configuration used for this work is the Common Research Model, which is a fullscale, cantilevered wing. The CRM is a modern single-aisle transport class aircraft configuration that was generated as an open geometry for collaborative research within the aerodynamics community. It has a wingspan of $192.8 \mathrm{ft}$, an aspect ratio of nine, a taper ratio of 0.275 , a leading edge sweep angle of $35^{\circ}$, and a break along the trailing edge at $37 \%$ of the semi-span (also referred to as a yehudi break) [3]. A traditional internal structure was developed in this work to use as a baseline for structural analysis. The wing box was defined to lie between $10 \%$ and $70 \%$ of the local chord.

The baseline wing box topology used for this work is shown in Figure 2 and consists of full-depth spars at the box leading edge, trailing edge, and one-third of the distance between the two. Thirty-seven straight ribs are evenly distributed from root to tip, each aligned with the airflow. Seven pairs of stringers (one on each skin) travel from root to tip: two pairs are evenly distributed between the leading edge spar and the inner spar, and five pairs between the inner and trailing edge spar. These stringers have a rectangular cross section, with a depth of 2.95 inches and a thickness of 0.18 inches. A full-depth rib stiffener exists at each stringer-rib intersection, each with a depth of 2.64 inches and a thickness of 0.18 inches.

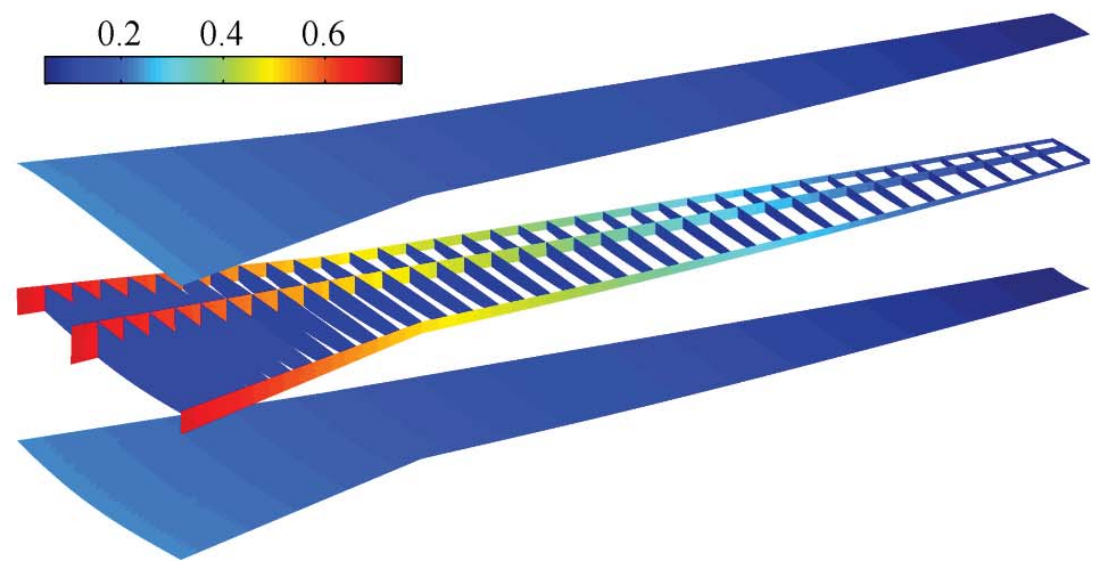

Figure 2. Baseline CRM structure used for tailoring studies: contour indicates local shell thickness (inches).

The thickness distribution of the ribs, spars, and wing skins is seen in the figure, and aluminum (2024-T3 alloy) was used throughout. Spars, ribs, and skins were modeled with higher-ordered triangular shell elements, stringers and rib stiffeners were modeled with beam elements, and the displacement degrees of freedom of all nodes at the wing-root of Figure 2 were fixed to zero. The inertial impact of leading and trailing edge control effectors were modeled as lumped masses, connected to the leading and trailing edge spars via un-weighted interpolation elements. 
Six $320 \mathrm{lb}$ masses were used along the inboard leading edge, and three additional of $240 \mathrm{lbs}$ outboard. Similarly, six $840 \mathrm{lb}$ masses and three $140 \mathrm{lb}$ masses were used along the trailing edge. These mass values were calculated by scaling data from a similar commercial transport.

The tow steering results of Section IV replace the metallic skins of Figure 2 with composite laminates, but the internal structure is kept the same as the baseline structure. The results of Section V change the metallic material properties and/or the thickness distribution of the skins, ribs, and spars. The studies in Section VI leave the material and overall thicknesses the same as the baseline, but alter the number, location, orientation, and curvilinearity of the ribs and spars. The structural weight and aeroelastic performance of each of these permutations is continually compared to the performance of the all-metallic baseline wing structure of Figure 2.

\section{Aeroelastic Modeling Procedures}

Starting with the outer mold line of the CRM [3], MATLAB scripts were used to generate PATRAN session files to populate the CRM outer mold line with a user-defined topology of ribs, spars, stringers, and rib-stiffeners (all of which may be curvilinear or straight). The resulting geometry was auto-meshed using CTRIAR elements (ribs, spars, and skins) and CBAR elements (stringers and rib stiffeners) to define the finite element model. A static aeroelastic analysis, buckling analysis, and a flutter analysis were then conducted in NASTRAN. MATLAB scripts were used to generate input files for the analyses and to extract the data from the NASTRAN output files to compare performance metrics and assess the aeroelastic tailoring concepts. Flat-plate aerodynamic paneling was utilized for both steady and unsteady air loads, with a $10 \times 10$ mesh of boxes for the inboard section of the wing (spanning from the wing root to the yehudi break), and a 10×40 mesh for the outboard wing section (spanning from the yehudi break to the wing tip). Finite element nodes located at intersections of the upper skins and ribs, or the upper skins and spars, were used to interpolate between the structural and aerodynamic meshes.

Static aeroelastic wing deformation was computed at specified angles of attack of $-2^{\circ}, 0^{\circ}, 2^{\circ}, 4^{\circ}$, and $6^{\circ}$, a Mach number of 0.85 , and an altitude of $35 \mathrm{kft}$. The resulting data set was distilled into structural weight, wing tip deflection/twist, and an aggregate stress metric (Kreisselmeier-Steinhauser (KS) function [19]), where low values are desirable. The line of centers of gravity from root to tip were also computed, along with the flexural axis, which is a locus of points along the wing at which, in response to an applied load at their locations, the wing deforms without a change in the local angle of attack [20]. Buckling eigenvalues were also computed for each deformed state and the corresponding buckling mode. This can be done for each of the aeroelastic trim cases, but only the extremes $\left(-2^{\circ}\right.$ and $\left.6^{\circ}\right)$ are typically of interest. A flutter analysis (p-k method) was then performed with 20 structural dynamic modes at a Mach number of 0.85 , and using the speed of sound at sea level, the velocity was computed and fixed. The dynamic pressure varied from 0 to $14.8 \mathrm{psi}$ and was divided into 250 increments by varying the flow density; zero-damping cross-over points indicate flutter.

\section{Tow Steered Composites}

There are numerous design variables that can be used to tailor composite skins through varying the fiber angles and stacking sequences. This section describes eight different scenarios, with increasing levels of tailorability and comparing more traditional laminates (tailored via number of plies at different angles) with tow steered composites (tailored via curvilinear fiber tow paths within each ply). Comparing these scenarios allows systematic evaluation of the effectiveness of different variables within composite skin design for carrying static and dynamic loads in an aircraft wing. The first four scenarios are for straight, non-steered laminates, globally varying the locations of the different tailored laminates on the wings: (1) same laminates for upper and lower skins; (2) different laminates for upper and lower skins; (3) each skin divided into 4 laminates from root to tip but the upper and lower skins are the same; and (4) each skin divided into 4 laminates from root to tip with upper and lower skins different. The next four scenarios follow the same approach, but also introduce the ability to steer the tow angles within the plane of the laminate instead of holding the fibers straight and parallel. Thus, the tow steered scenarios are: (5) same laminates for upper and lower skins, steered from root to tip; (6) different laminates for upper and lower skins, steered from root to tip; (7) five control points steered from root to tip but same laminates on upper and lower skins; and (8) five control points steered from root to tip with different laminates on upper and lower skins.

An example composite design string is defined as $\{3,1,4\}$, where the first digit is the number of $\pm 45^{\circ}$ stacks $\left(N_{ \pm 45^{\circ}}\right)$, the second digit is the number of $90_{2}^{\circ}$ stacks $\left(N_{2 \times 90^{\circ}}\right)$, and the third digit the number of $0_{2}^{\circ}$ stacks $\left(N_{2 \times 0^{\circ}}\right)$. Each variable creates twice the number of plies (for a balanced laminate), and the total number of plies is further doubled to create a symmetric laminate. The design string $\{3,1,4\}\left(N_{ \pm 45^{\circ}}=3, N_{2 \times 90^{\circ}}=1\right.$, and $\left.N_{2 \times 0^{\circ}}=4\right)$ thus has 32 total layers. Because the depth of the wing box is much larger than the thickness of each skin, the in-plane stiffness dominates the bending stiffness (for global wing deformations), making the details of the stacking sequence 
unimportant. Three design variables were used to define each laminate, where the lower bound of $N_{ \pm 45^{\circ}}$ is 1 , but both $N_{2 \times 90^{\circ}}$ and $N_{2 \times 0^{\circ}}$ are allowed to become 0 . The upper bound of each variable is 4 . For this study, the $0^{\circ}$ orientation is parallel to the flow-direction, along the wing's root-chord axis.

Because the effect of the fiber steering paths depend heavily upon the underlying laminate details, attention is first paid to the design of un-steered laminate wing skins, so that steered laminate performance may be put into context. A multi-objective genetic algorithm (GA) [21] was used to locate the Pareto front between minimum weight, minimum KS function (i.e., low aeroelastic stresses), and maximum flutter dynamic pressure. Though GA is an optimization tool, its main utility here is to provide a large distribution of sampling points across the highperforming portions of the design space. The designs with the lowest stress (i.e. lowest KS function) are shown in Figure 3, for each of four scenarios: the same laminate for both upper and lower wing skins (3 design variables), separate laminates for the skins (6 variables), each skin divided into 4 laminates from root to tip (12 variables, with the upper and lower skins the same), and eight total laminates across the wing (24 variables, with upper and lower skins different). The wing weight and flutter dynamic pressures shown were normalized by the values of the baseline structure in Figure 2. Thus to obtain the stated objectives of reduced wing weight, the stress (KS) and weight values should be minimized while the flutter speed should be maximized.
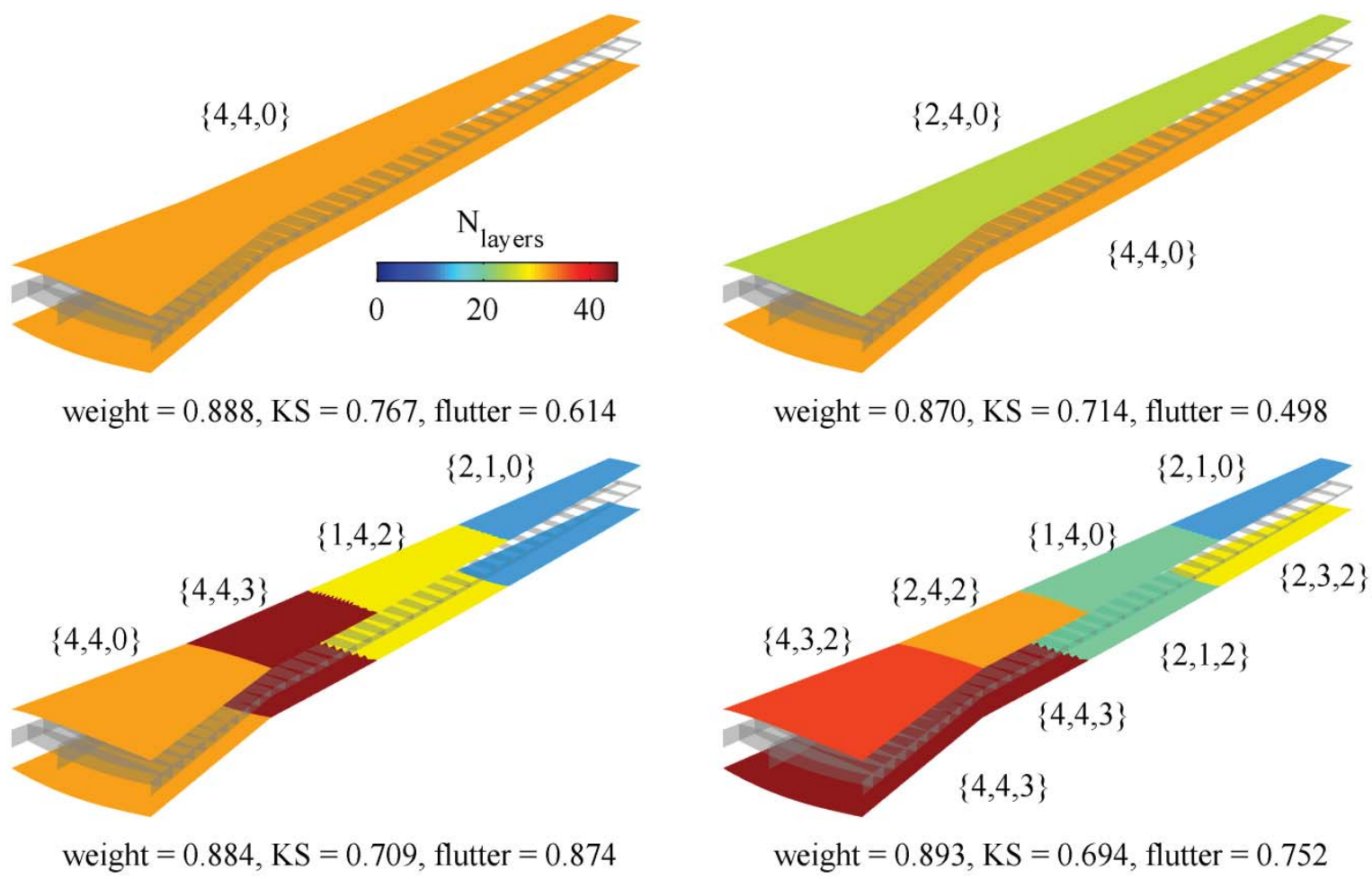

$$
\text { weight }=0.884, \mathrm{KS}=0.709 \text {, flutter }=0.874
$$

$$
\text { weight }=0.893, \mathrm{KS}=0.694 \text {, flutter }=0.752
$$

\section{Figure 3. Designs with the lowest aggregate stress (KS function) under various spatial laminate parameterizations.}

Amongst the best designs (lowest KS values) for each of the four un-steered scenarios, the KS stress function progressively dropped from 0.767 to 0.694 as the degree of tailorability increases, as would be expected. The lowest stress did not always trend towards the highest or lowest weight. It is noted that the best flutter design was always found to be the heaviest 48 -ply laminate $\{4,4,4\}$, (i.e., there is a very strong trade-off between wing weight and flutter, unlike the KS-weight trend of Figure 3). This indicates that increasing the number of design variables enables tailoring of the stress, but not necessarily the weight or the flutter speed.

Progressing on from un-steered laminates to the additional variable of tow steering the fiber paths within the laminate, a simple full-depth tow steering concept is used here, taken from Ref. [5]. A steering angle is specified at the wing root $\left(\Theta_{1}\right)$ and the tip $\left(\Theta_{2}\right)$, with a linear variation in between: this results in a curvilinear tow path. Then, the core laminate, as defined by $N_{ \pm 45^{\circ}}, N_{2 \times 90^{\circ}}$, and $N_{2 \times 0^{\circ}}$, is rotated with the steering path, where $90^{\circ}$ fibers (if any) are those that will remain parallel to the path at every location through the wing skin, $0^{\circ}$ fibers are perpendicular to 
the path, etc. A steering angle $\left(\Theta_{1}\right.$ or $\left.\Theta_{2}\right)$ of $90^{\circ}$ means that a laminate is locally un-steered, and an angle of $60^{\circ}$ would align the $90^{\circ}$ fibers (if any) approximately with the leading edge of the swept CRM planform.

Though better results were obtained (as seen by the progression in Figure 3), this scenario uses $\{4,4,0\}$ for the upper and lower skins to directly compare the effects of steered versus un-steered laminates. The KS values are shown in Figure 4 as a contour plot, where lower values directly correlate to lower stresses throughout the wing structure, when $\Theta_{1}$ and $\Theta_{2}$ are independently swept from $30^{\circ}$ to $150^{\circ}$. Steering patterns are shown for various data points along the perimeter of the plot moving in a counter-clockwise direction, starting at the lower left corner: $\left[30^{\circ}\right.$ $\left.30^{\circ}\right],\left[90^{\circ} 30^{\circ}\right],\left[150^{\circ} 30^{\circ}\right],\left[150^{\circ} 90^{\circ}\right],\left[150^{\circ} 150^{\circ}\right],\left[90^{\circ} 150^{\circ}\right],\left[30^{\circ} 150^{\circ}\right]$, and $\left[30^{\circ} 90^{\circ}\right]$. It is further noted that each of these designs has the same weight (equal to the normalized value of 0.888 from the un-steered structure in Figure 3): tow steering merely re-orients existing material and is not additive.

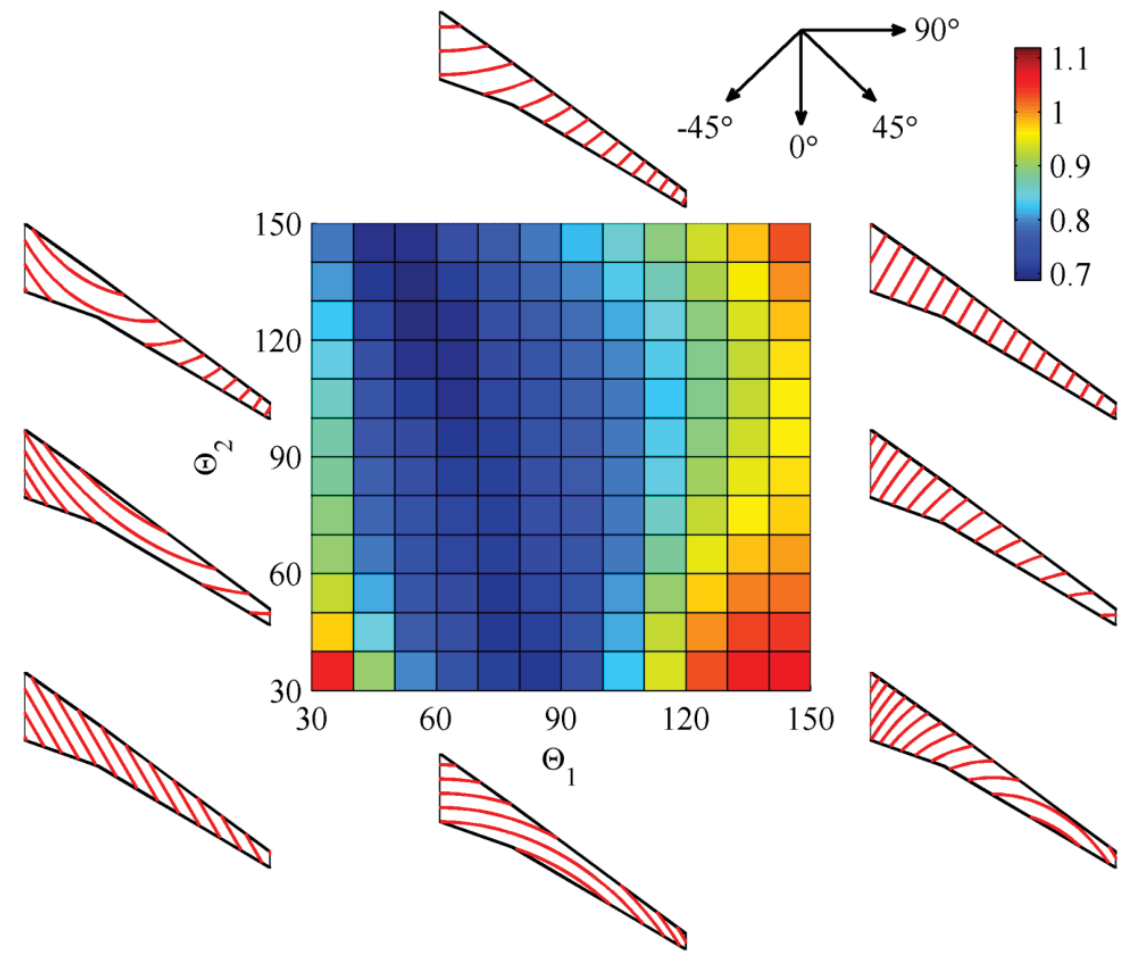

Figure 4. Aggregate stress function (KS) for different $\operatorname{root}\left(\Theta_{1}\right)$ and tip $\left(\Theta_{2}\right)$ steering: $N_{ \pm 45^{\circ}}=4, N_{2 \times 90^{\circ}}=4$, and $N_{2 \times 0^{\circ}}=0$ for both the entire upper and the lower skin laminate.

The data point at $\left[90^{\circ} 90^{\circ}\right]$ which corresponds to the center of the figure is entirely un-steered and gives the exact same performance as the structure in the upper left of Figure $3(\mathrm{KS}=0.767)$. Any design along the line $\Theta_{1}=\Theta_{2}$ (the diagonal) is un-steered in the sense that there are no curvilinear fibers, but the entire stacking sequence is rotated uniformly from root to tip, as drawn in the lower left $\left[30^{\circ} 30^{\circ}\right]$ and upper right $\left[150^{\circ} 150^{\circ}\right]$ subplots. Only when $\Theta_{1}$ and $\Theta_{2}$ are unequal do curvilinear paths emerge, the best of which (in terms of the lowest KS value, and hence the lowest stresses) is $\left[50^{\circ} 150^{\circ}\right]$, which has a performance improvement of $9.6 \%$ over the un-steered case $\left[90^{\circ} 90^{\circ}\right]$. As may be expected, this high-performing steering path is nearly aligned with the CRM leading edge at the wing root, and perpendicular to it at the wing tip (presumably due to fibers aligning with principal stress directions of the fundamental load paths along the wing).

Similar data can be obtained for the dependence of flutter upon the steering path, but is not shown here. The best flutter point is located at $\left[150^{\circ} 150^{\circ}\right]$ (a $52.3 \%$ increase in the flutter speed over the un-steered case), which uniformly rotates all the fibers counterclockwise by $60^{\circ}$, nearly perpendicular to the leading edge. Though some benefit to flutter via curvilinear tow paths will be found below, on the whole, the flutter benefits were found to be less than those associated with aeroelastic strength (KS). This may be simply due to the fact that curvilinear steering is a spatially-local tailoring scheme with a minor impact upon a global flutter mode. The wing stresses, on the other hand, are a local metric (albeit due to global wing deformations), which can bear greater improvement from steering. 
For the next scenario, different steering patterns were considered for the upper and lower skins, where the core laminate for both skins still use the design string of $\{4,4,0\}$, which was the optimal solution for the un-steered laminates from Figure 3. The root steering angle $\Theta_{1}$ is fixed at an arbitrary value of $90^{\circ}$ (to reduce the design space), while the tip steering angles $\Theta_{2}$ for the upper and lower skins are independently varied through $10^{\circ}$ increments. Results for the KS function are given in Figure 5. As before, select steering patterns are drawn for various designs along the perimeter, where the red lines are the paths along the upper skin, and the blue lines are for the lower skin. These paths are identical at the wing root, of course, because $\Theta_{1}$ is fixed at $90^{\circ}$ for both. The design at $\left[90^{\circ} 90^{\circ}\right]$ is entirely un-steered, as before and identical to the first scenario in the upper left of Figure 3.

Any design in these figures along the unit slope (US $\Theta_{2}=\operatorname{LS} \Theta_{2}$ ) has no difference in the steering patterns between the upper and lower skins. Any design along the vertical line US $\Theta_{2}=90^{\circ}$ has no steering in the upper skins, and any design along the horizontal line LS $\Theta_{2}=90^{\circ}$ has no steering in the lower skins. The design with the lowest stresses (i.e. lowest KS function) is found at $\left[140^{\circ} 30^{\circ}\right]$, which presents a $13.5 \%$ reduction as compared to the un-steered $\left[90^{\circ} 90^{\circ}\right]$ case. This is superior to what was found with identical upper and lower tow steered skins (in Figure 4); presumably even better designs exist if one drops the restriction that $\Theta_{1}=90^{\circ}$, and conducts an exhaustive search through the 4-dimensional space. The best flutter result (again not shown here) is found at $\left[30^{\circ}\right.$ $30^{\circ}$ ], a design which improves the flutter point by $40.4 \%$ as compared to the un-steered case. This optimal flutter $\left[30^{\circ} 30^{\circ}\right]$ design has no differences in the steering between upper and lower skins, and a better, entirely un-steered result, can be found in the previous scenario.

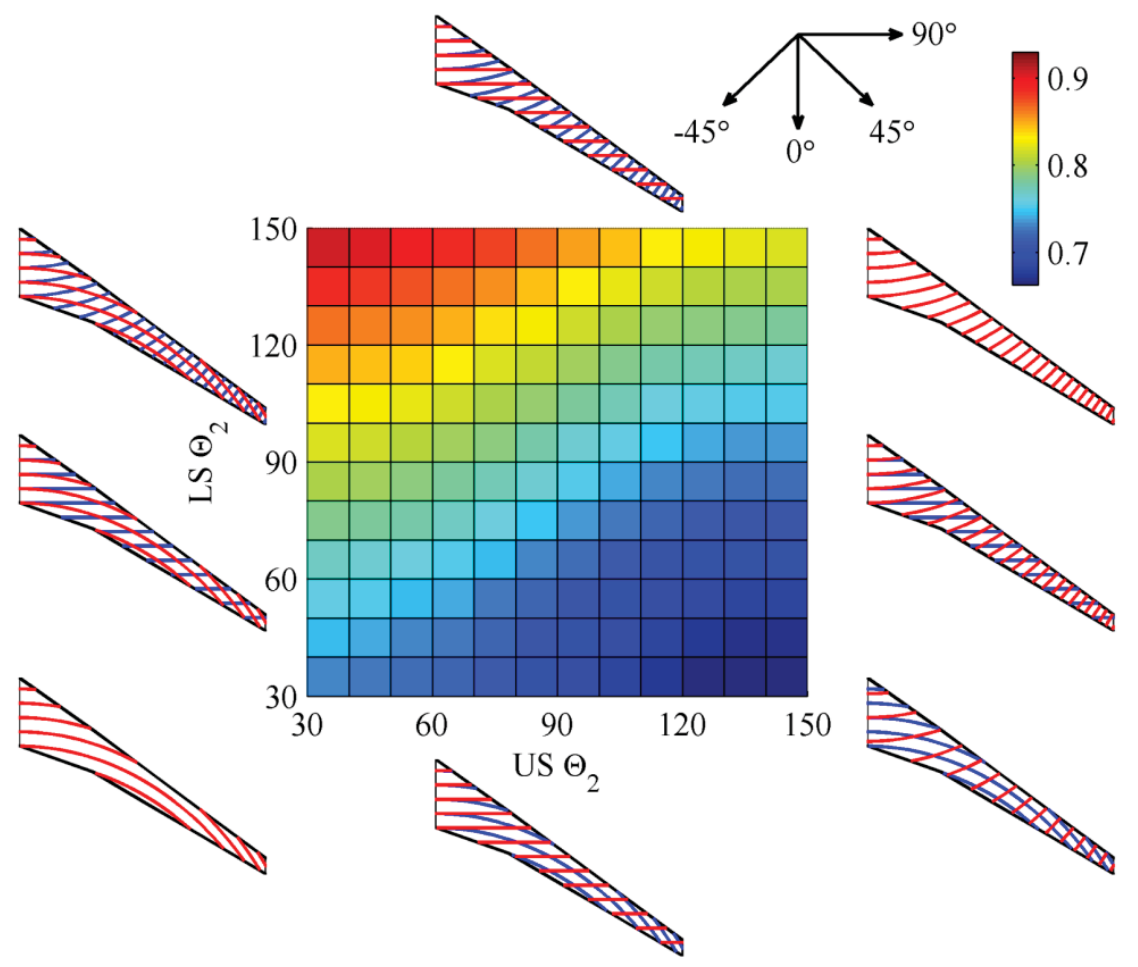

Figure 5. Aggregate stress function (KS) with a root $\Theta_{1}$ of $90^{\circ}$, and different upper skin tip (US $\left.\Theta_{2}\right)$ and lower skin tip (LS $\Theta_{2}$ ) steering: $N_{ \pm 45^{\circ}}=4, N_{2 \times 90^{\circ}}=4$, and $N_{2 \times 0^{\circ}}=0$ for both the entire upper and the lower skin laminate.

The previous results used steering concepts with only two control points (wing root and tip) and a linear variation in between. The final two scenarios in this section use five control points and a piece-wise linear variation along the span, with identical (five continuous design variables) and different (ten continuous design variables) upper and lower skins. The starting point for both was a Pareto-optimal design in terms of weight, KS and flutter point, where each wing skin was broken into four segments along the span (as shown in the inset in the lower right corner of Figure 6). A steering control point (design variable) was placed at the edge of each segment. This design shows the expected taper (i.e., thinner shell at the tip than at the root) towards the wing tip, and the inner two panels 
happen to have the same thickness (though different laminates). Figure 6 shows the results from the genetic algorithm that was used to find the 2-D Pareto front between KS and flutter.

An asterisk in Figure 6 marks the performance of the un-steered structure, which is dominated by both Pareto fronts, (i.e., it has higher stresses and a lower flutter point than the fronts). For both sets of GA results, steering patterns are drawn into the plot along various regions of the fronts; for the case where upper and lower skin steering is distinct, red lines correspond to the upper skin and blue to the lower skin. The results of Figure 6 provide the only results in this work that show a flutter-based benefit to curvilinear tow steering. The steering path for these highflutter speed designs do not take strong advantage of the higher-ordered curves available to it (i.e., the steering angle variation from wing root to tip is weakly nonlinear), and so the flutter-benefit seen here, but not above, may be largely due to the tapered underlying laminate.

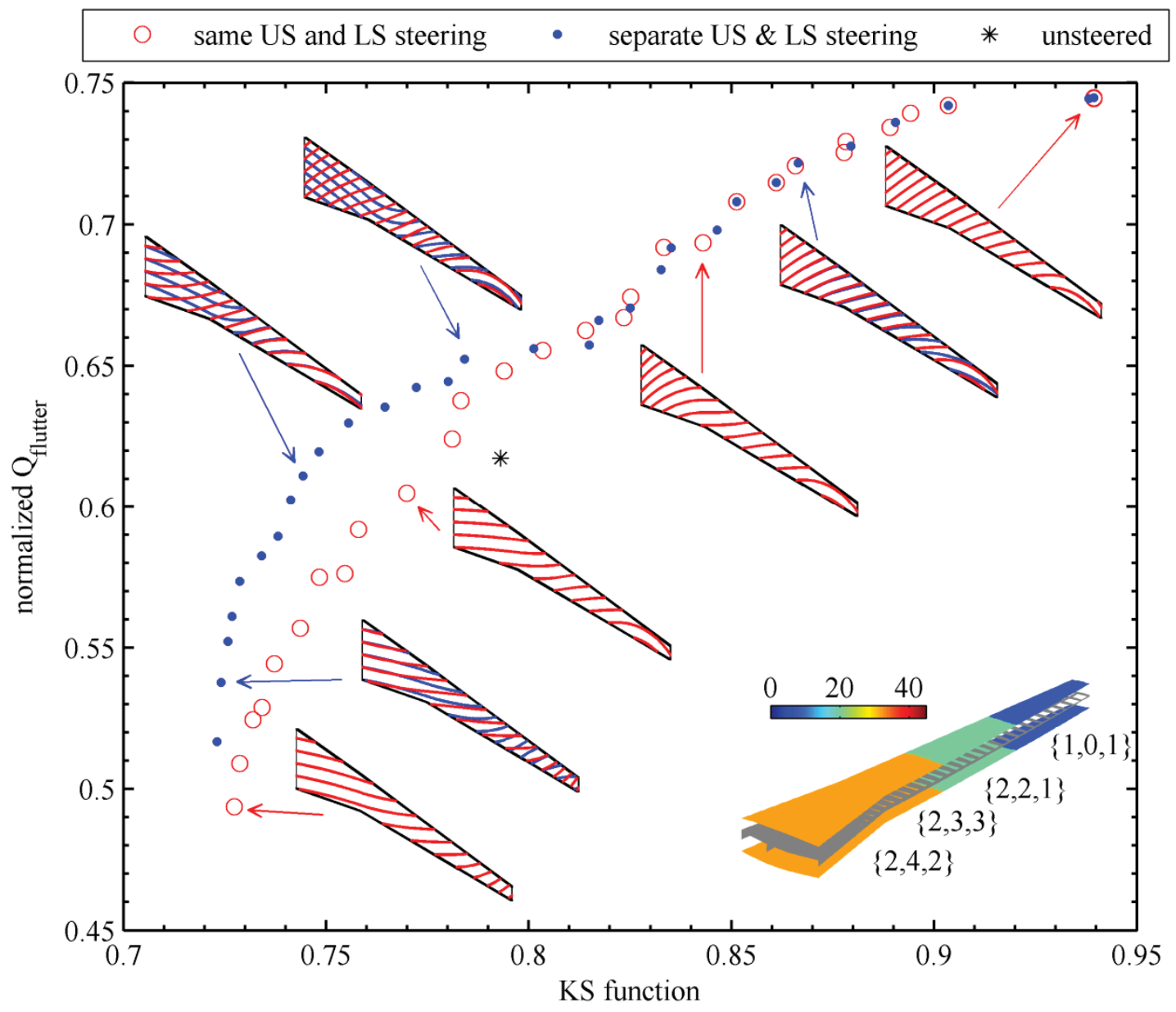

Figure 6. Pareto front: multiple laminates per skin with higher-order steering concepts.

Furthermore, there is little separation between the two Pareto fronts in the high-flutter point region, which does echo previous results: flutter resistance has only minor benefit from separate steering strategies between the upper and lower skins. Of course, this conclusion may be shifted by a situation where different core laminates are used in the two skins. In the low-stress (and thus low flutter speed, due to the strong trade-off) region, however, there is noticeable benefit to using different steering strategies between the upper and lower skins. By allowing separate steering, the flutter point may be improved with no penalty in wing stresses, or vice-versa. It is also noted that these designs with separate steering and low stresses (designs in the upper left) are the only ones that show a noticeable nonlinear steering angle variation from root to tip. Significant undulations are particularly seen in the lower skins. It may be that increasing the rib separation within the wing box, and thus increasing the level of local panel vibration/deformation seen during aeroelastic behavior, may increase the need for significant variations in the local steering angle along the wing. Of course, the core laminate chosen for this exercise may heavily bias these 
conclusions. Additional details regarding the work presented in this section will be published in the near future as a NASA Technical Memorandum.

\section{Material and Thickness Grading}

Recent advances in manufacturing methods allow for continuous grading of materials and thicknesses from one level to another. In this section, the different scenarios investigated included keeping the alloy the same as the baseline CRM model but tailoring the thicknesses of the different components, and functionally grading from one material composition to another to locally tailor material properties within the structure. For all cases, the structural arrangement of skins, ribs, spars, and stiffeners from the CRM baseline was held constant.

Thickness variables were allowed to range from a minimum of 0.05 inches to a maximum of 0.75 inches. Compositional gradients were accomplished by blending from an aluminum alloy to aluminum with silicon carbide particulates ( $\mathrm{Al}+\mathrm{SiC}$ : specifically, 6092/SiC/17.5p), where the material fraction was specified at every location along the wing. The two materials have nearly the same density, but $\mathrm{Al}+\mathrm{SiC}$ has $44 \%$ higher modulus and $57 \%$ higher yield stress than $\mathrm{Al}$, enabling spatial tailoring of material properties that would typically be uniform. In addition, for this combination of materials, local stiffness changes are possible without affecting the local mass, unlike stiffness changes due to thickness variations. The material fraction variables, which vary from zero (100\% aluminum) to one (100\% $\mathrm{Al}+\mathrm{SiC})$, and the thickness variables, which range within the prescribed bounds previously mentioned, are assigned to the four corners of the wing box planform. A bilinear interpolation is used for the interior, enabling linear tailoring in two directions (from root to tip and from the front spar to the rear spar).

For simplicity, the tailoring studies were separated into skin-only tailoring, spar-only tailoring, and rib-only tailoring studies. When not being tailored, the material and thickness variations were fixed at the same values used in the baseline CRM structure, whose thickness distribution is shown in Figure 2, using aluminum throughout. A multi-objective genetic algorithm [21] was used to find the Pareto front between the KS function (static aeroelastic stresses) and the normalized flutter dynamic pressure, where lower KS values and higher flutter point values are desired. The algorithm was forced to satisfy a weight equality constraint, such that all designs across the Pareto fronts have the same weight as the baseline.

For the tailoring of the wing skins, three studies were conducted. First, the material was held constant as uniform aluminum alloy, and the thickness design variables were altered to locate the Pareto front. Secondly, the thicknesses were fixed at their baseline CRM values, and the compositional material fraction distribution was varied in the GA. Finally, both thickness and material composition were allowed to change simultaneously. This process was repeated for the spars (three total), and again for the ribs, resulting in 9 total Pareto fronts, as seen in Figure 7. The performance of the baseline design is also provided in this figure. Tailoring of the wing skins clearly has the biggest impact upon the aeroelastic performance, partially due to the large acreage of these structures. Contrastingly, spar tailoring is more limited in terms of its ability to impact the aeroelastic performance beyond the baseline's, and rib tailoring has very moderate improvements.

The Pareto front in Figure 7 with thickness tailoring of the wing skins only (their material is fixed as $\mathrm{Al}$ ) is plotted with the red circles. The migration of thickness design variables for four selected locations along the front is depicted in Figure 8. In general, peak thickness begins at the leading edge of the root for designs with low aeroelastic stresses, shifts towards the root trailing edge, and finally to the leading edge of the wing tip for designs with improved flutter resistance. This is indicative of the general trade-off between static and dynamic aeroelastic phenomena, as only the latter will be strongly affected by (and see any improvement due to) added material at the wing tip.

For the lowest stress design (left-most extreme of the Pareto front), high thickness at the leading edge root is clearly valued, but the other design variables are already near the lower bound, and so additional thickness at the root cannot be obtained without violating the constant weight constraint. This is a clear attempt to globally stiffen the overall wing structure for stress reduction, but is not necessarily an aeroelastic tailoring strategy. The migration of the flexural axis [20] is very minor towards the left end of this front, and actually moves in a direction so as to slightly decrease the bend-twist wash-out (load-alleviation), rather than the desired increase. On the opposite end of the front (designs with high flutter dynamic pressures, i.e. high flutter resistance), very high thickness is desired at the leading edge tip, but is ultimately unable to reach the upper bound due to the weight restrictions. This follows the well-known trend of pushing the center of gravity forward for improved flutter resistance [22].

Even though the genetic algorithm was given the opportunity to tailor the upper and lower skins separately, the two distributions as seen in Figure 8 are fairly similar. This is in stark contrast with the previous tow steering results (Figure 6, for example) where some value is found in disparate tailoring schemes for the upper and lower wing skins, for low-stress designs (high-flutter point designs found little benefit). 


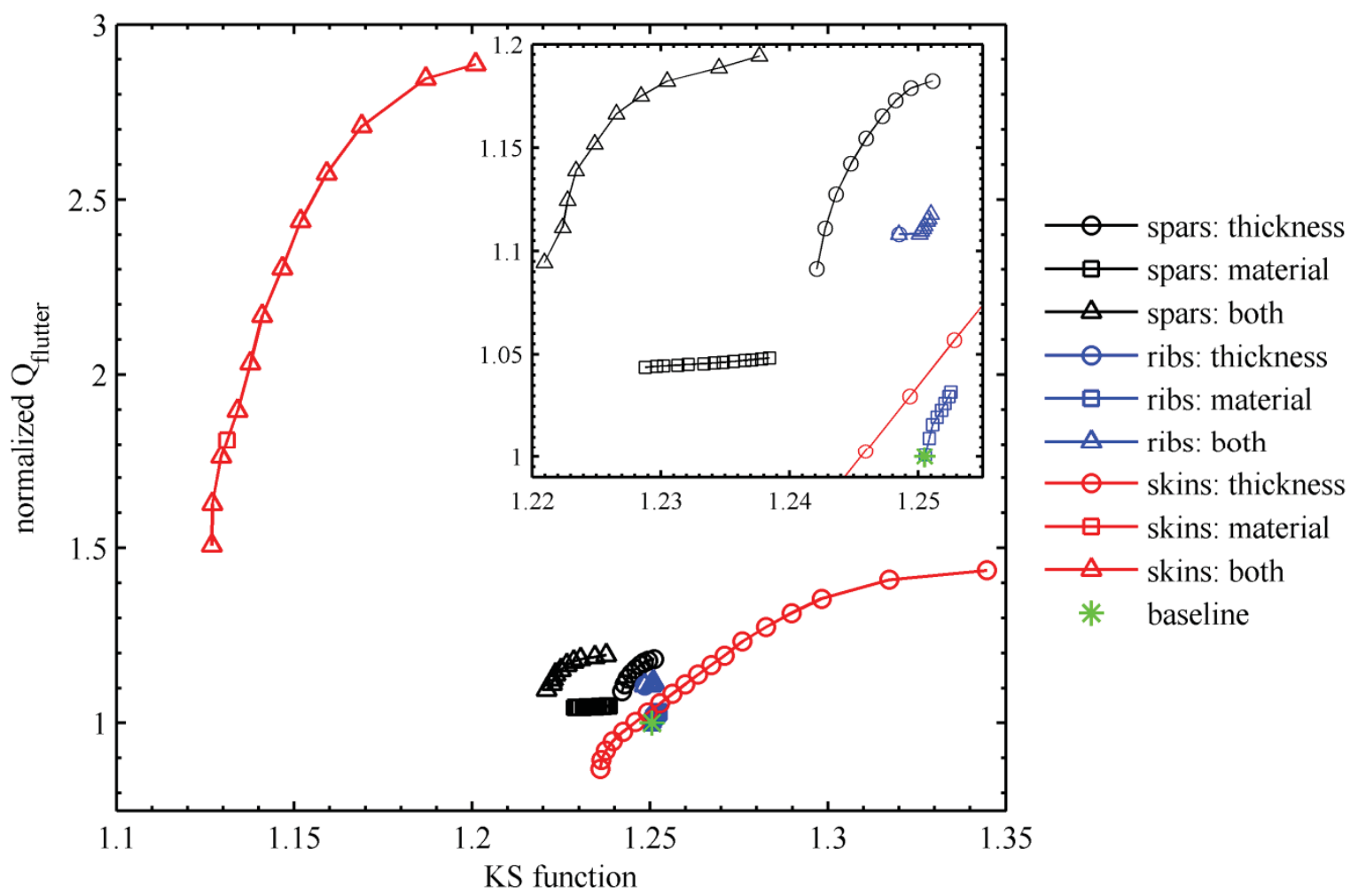

Figure 7. Pareto fronts between aeroelastic flutter (higher values indicate better flutter resistance) and KS function (lower values indicate lower stresses) for various grading strategies: all designs have the same weight as the baseline CRM.

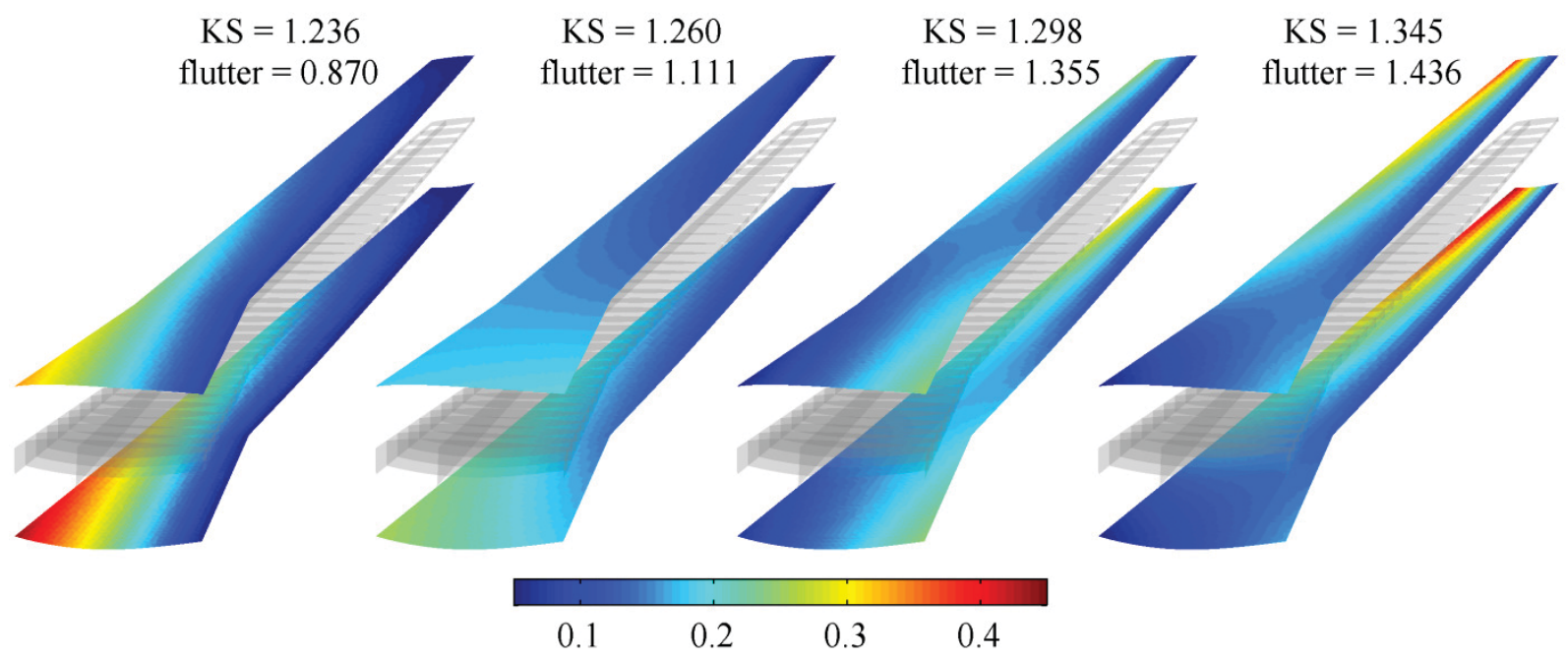

Figure 8. Skin thickness distribution for select designs across the Pareto front of Figure 7, without material tailoring. All designs have the same weight as the baseline CRM.

Next, the thickness distribution of the skins was set equal to the baseline CRM values and the material fraction variables of the upper and lower skins were allowed to change. The resulting Pareto front (Figure 7), shown as red squares, has devolved into a single point. Each material design variable has been forced to unity: the structure is 
made entirely of the stiffer of the two materials, $\mathrm{Al}+\mathrm{SiC}$. This is a trivial answer of course, in the sense that it is usually expected for an increase in stiffness with no associated weight penalty (as the density of the two materials is the same) to have a favorable impact on many aero-structural metrics. The goal of this study is to identify situations where spatial material grading may be used, where a structure may benefit from a localized reduction in the stiffness/modulus, but this is not observed here (potentially limited by the bilinear grading distribution).

The next step was to allow for simultaneous changes in both the material and thickness distribution of the upper and lower skins. The resulting Pareto front is also given in Figure 7 as red triangles, and shows a clear and substantial improvement over the thickness-only Pareto front discussed above: a 9.8\% drop in the lowest KS value and a near-doubling of the best flutter speed. As before, however, each of these structures is entirely composed of the stiffer $\mathrm{Al}+\mathrm{SiC}$ material. Only thickness variations are used to form the Pareto front, and the trends of each thickness variable are very similar to Figure 8 . For this wing configuration, and this $\mathrm{Al}-(\mathrm{Al}+\mathrm{SiC})$ material combination, functionally graded materials in the wing skins is not found to provide any benefit.

The above exercise was repeated for the wing spars, holding the material and thickness distribution of the skins equal to that of the baseline. As noted, the performance improvements available from this study are far less than what could theoretically be obtained from tailoring the wing skins, but tailoring of a spar (or rib, or stringer) is perhaps more feasible given the current manufacturing state-of-the-art. True material grading is seen to be beneficial for spar structures, however, for both the material-only Pareto front (black circles in Figure 7), as well as the combined thickness/material front (black triangles in Figure 7). Thickness and material distributions from 3 select locations along this latter front are shown in Figure 9. This combined front provides a leftward shift as compared to the thickness-only behavior: the stresses (KS function) are decreased, but flutter behavior is relatively constant.

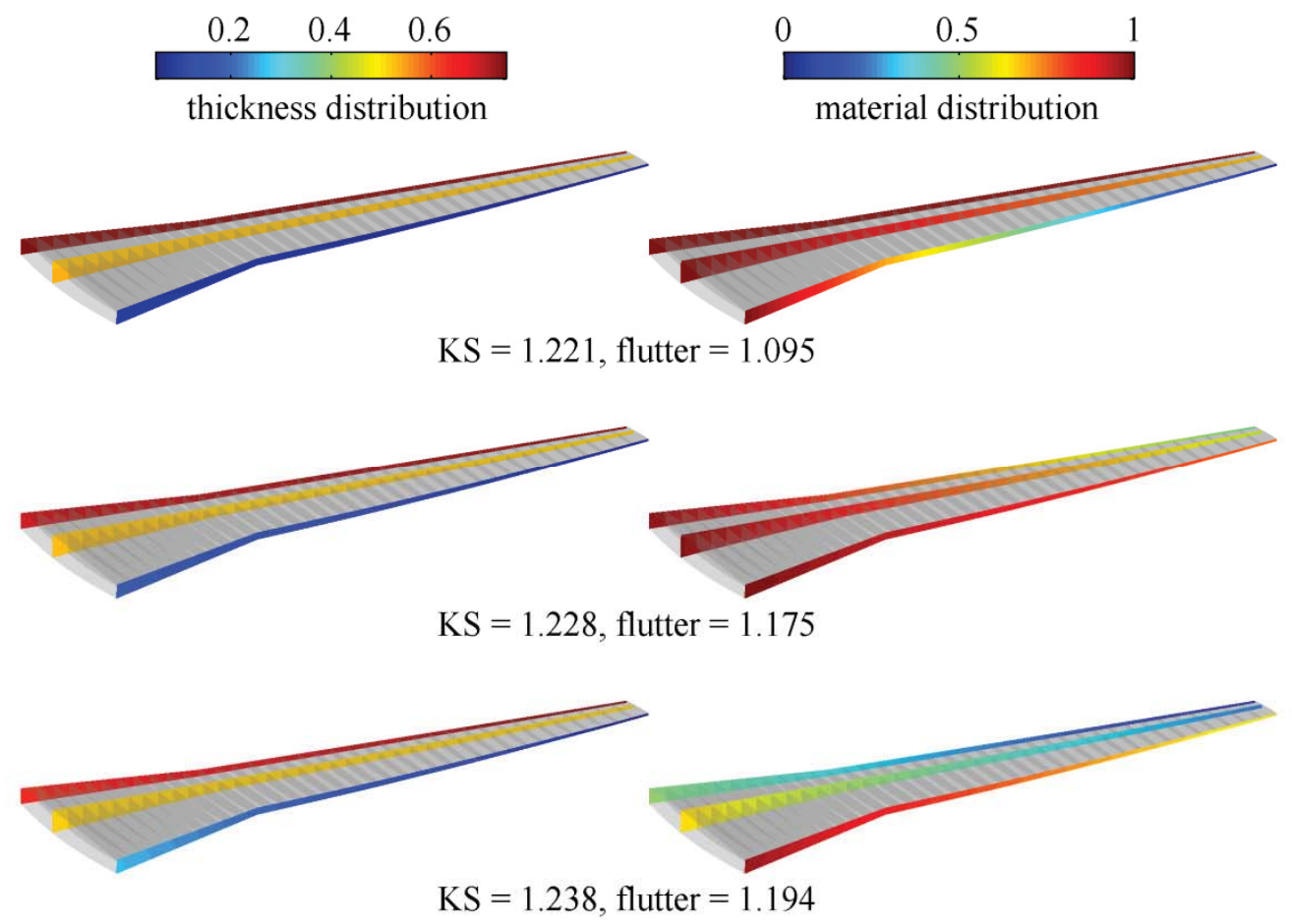

Figure 9. Spar thickness (left) and material fraction (right) distributions for select designs across the Pareto front.

For the design with the best aeroelastic strength, both leading edge thickness design variables are nearly equal to the upper bound of 0.75 inches, and both trailing edge points are nearly equal to the lower bound of 0.05 inches. It is noted that for the wing skin tailoring study, it was impossible for any of the thickness variables to approach this upper bound (even if the other 3 variables were set to the lower bound) without violating the constant mass constraint. For spar tailoring, strictly due to the geometry of the problem, it is possible for two variables to reach this upper limit and still retain the required weight (equal to the baseline). 
The stiffer $\mathrm{Al}+\mathrm{SiC}$ is mostly used at the trailing edge root across the entire Pareto front, but the high-strength design (top of Figure 9) does see some benefit to using the weaker material at the trailing edge tip. This may follow the thickness patterns, where flexibility is built into the trailing edge of the wing, but is largely unexpected due to the non-importance of material stiffness and yield stress at the tip, as opposed to inertial quantities. For high-flutter speed designs (bottom of Figure 9), a substantial portion of the leading edge spar has been reverted to the weaker material. The reason behind this shift is presumably due to a design synergy with the thickness distribution, which has made the leading edge very heavy in an attempt to shift the overall center of gravity (thus separating the vibration modes and improving the flutter resistance). A reduction in the local modulus of these thick members may further improve this modal separation, from a stiffness (rather than inertial) standpoint.

For tailoring of the wing ribs, as seen in Figure 7, both thickness (blue circles) and material tailoring (blue squares) only moderately improve the aeroelastic performance over the baseline structure. This is because rib structures do not support significant bending or torsional loads, as opposed to wing skins and spars; one of their significant functions is to maintain the airfoil shape of the outer mold line [23]. When thickness-only tailoring was used, the Pareto front devolves to a single point (blue circles): three thickness variables are set to the lower bound of 0.05 inches, and (to satisfy the constant weight constraint), the leading edge tip is set to 0.5 inches. This forwardmass design has obvious advantages from a flutter perspective (a 10.8\% increase over the baseline design), and presumably has no detrimental impact on the stress distribution.

Material grading of the ribs only improved the flutter resistance by $3.2 \%$, as shown in the left column of Figure 10. Each material variable monotonically increases across this Pareto front until it reaches the upper bound, with $100 \% \mathrm{Al}$ for the design with the lowest stress (a non-intuitive result) and 100\% $\mathrm{Al}+\mathrm{SiC}$ for that with the highest flutter speed. Finally, both material and thickness tailoring parameters are considered simultaneously, shown on the right of Figure 10.

The result when both thickness and material are graded is a combination of the previous two results. Thickness remains set at the distribution discussed above, across the entire Pareto front. Whereas the material-only grading found some benefit to using the weaker material along the leading edge for low-stress wing structures (before switching over to the stiffer material), the stiffer material is used for every design in this case. Trailing edge trends are the same, though weaker, and less impacted by the upper and lower bounds. An $11.8 \%$ peak improvement in flutter resistance is ultimately available over the baseline, with a trivial reduction in stress. As may be expected, the main utility in rib tailoring is inertia-based for improvements in flutter. Even the best designs in Figure 10, however, may be too thin in certain locations to withstand crushing (Brazier) loads [23], or to adequately maintain the OML of the flexible wing surface, and so the moderate demonstrated benefits may be negated by these (unmodeled) metrics. Additional details regarding these thickness and material grading results will be published as a NASA Technical Memorandum.
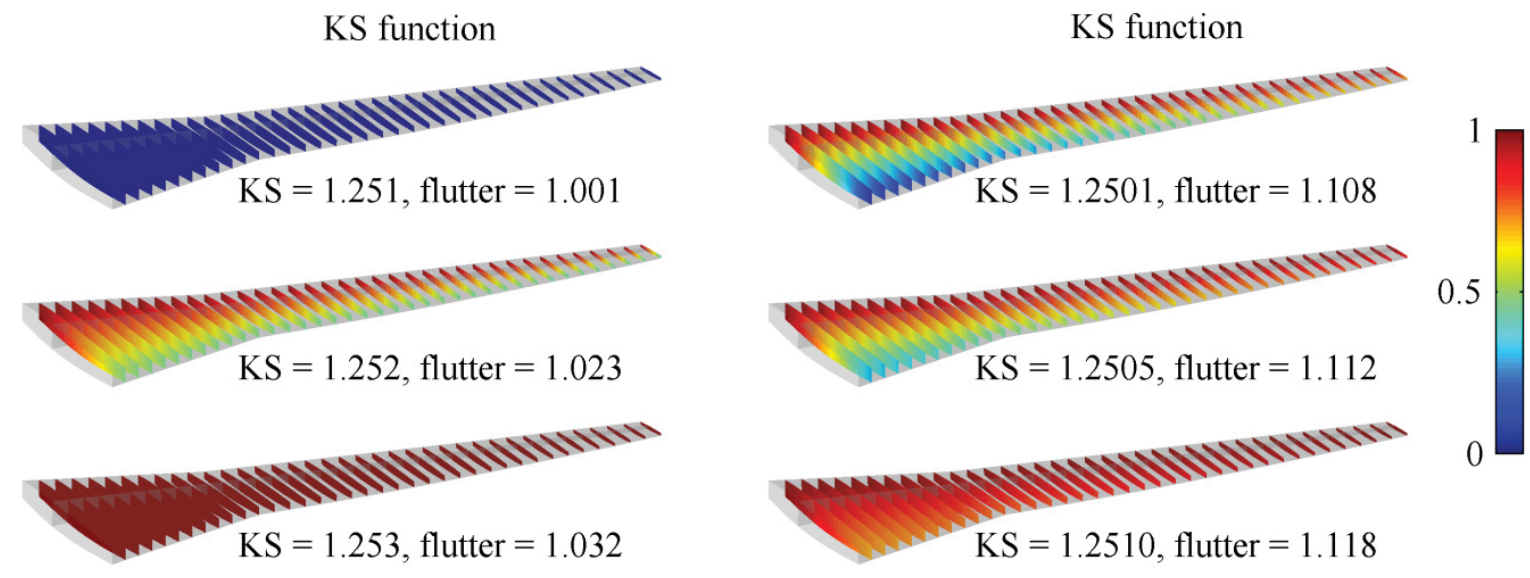

\section{Figure 10. Rib material distributions along the Pareto front when thickness is (right) and is not (left) tailored.}

\section{Curvilinear Rib and Spar Placement}

In addition to new materials and manufacturing methods that could tailor the structures to reduce weight without adversely affecting stress or flutter speed, structural approaches were considered. Twelve parametric 
studies were performed on the baseline CRM wing's internal structure, leaving the material and overall thicknesses the same as the baseline, but altering the number, location, orientation, and curvilinearity of the ribs and spars. A brief description of the studies is included in Table 1; the last column refers to designs highlighted in Figure 11. The first five studies modified the stringers and inner spar, keeping the baseline rib configuration constant. The next six studies modified the ribs, while keeping the baseline spar/stringer configuration constant. The final study combined the resulting designs from the spar-only and rib-only studies to seek further performance improvements and weight reductions.

In these studies, the geometry of a few specific structural members was fixed, including the front and rear spars, one rib at the wing yehudi break, one rib at the wing tip, and the skins. The internal rib and spar structure of the baseline CRM was parameterized using the Linked Shape Parameterization [24], which defines the structural members (either ribs or spars) of a wing section using b-splines. Modifications to the parameterization were made to allow straight ribs within a wing section to be reoriented at a new angle and to enable selected spar members to be removed and modeled as a pair of stringers instead. The number of structural members along with their location, orientation, curvature (or lack of curvature if straight) were modified using 26 design parameters; an additional vector of eight parameters defined the spanwise members as spars or stringers.

The previous two tailoring studies (tow steering and thickness/material grading) used the same mesh for every evaluation, whereas here, the wing box mesh had to be regenerated for each design permutation, making these studies more computationally expensive. It was not uncommon that PATRAN would have complications with creating geometry or meshing a design; this often occurred when the alignment of two structural members within a design caused highly skewed elements. Therefore, it was advantageous to study as many designs as possible to help minimize the effect of missing data points (gaps in the design space).

Table 1. Description of the parametric studies performed on the spars and ribs.

\begin{tabular}{|c|c|c|}
\hline Study & Investigations & $\begin{array}{l}\text { Designs in } \\
\text { Figure } 11\end{array}$ \\
\hline & Spar studies & \\
\hline 1 & Number of straight spars and their location & \\
\hline 2 & Curvature of inner spar (higher degree of curvature than study 3 ) & $a, b$ \\
\hline 3 & Curvature of inner spar (lower degree of curvature than study 2) & $\mathrm{c}$ \\
\hline 4 & Curvature of stringers only (inner spar removed) & $\mathrm{d}, \mathrm{e}$ \\
\hline \multirow[t]{2}{*}{5} & $\begin{array}{l}\text { Varying stringer curvature separately in inboard and outboard (inner spar } \\
\text { removed) }\end{array}$ & \\
\hline & Rib studies & \\
\hline 6 & Number of straight ribs & \\
\hline 7 & Rotating straight ribs (inboard and outboard orientations the same) & $\mathrm{g}$ \\
\hline 8 & Rotating straight ribs (only outboard orientation modified) & \\
\hline 9 & Rib curvature (same curvature for inboard and outboard) & $\mathrm{h}, \mathrm{i}$ \\
\hline 10 & Study 9 but with inner spar removed & $\mathrm{j}, \mathrm{k}, \mathrm{l}$ \\
\hline \multirow[t]{2}{*}{11} & Rib curvature (different curvature for inboard and outboard) & \\
\hline & Combined studies & \\
\hline 12 & Combines spar/stringer designs (studies 1-5) with rib designs (studies 6-11) & $\mathrm{m}, \mathrm{n}$ \\
\hline
\end{tabular}

To thoroughly explore the design space without using a time-intensive optimization approach, a full factorial approach [25] was used when applicable (especially on the spar/rib curvature parameters). The approach to these studies was first to learn which changes in the internal structure design have the greatest effect on both increasing the wing's flutter resistance and in decreasing its weight. Afterward, the remaining evaluation metrics (such as tip deflection, tip twist, location of the flexural axis, buckling resistance, and location of the center of gravity) were considered to determine additional trends and correlations to provide new insights into the design of aeroelastically tailored wings.

By varying the spar, stringer, and rib configurations, some designs were created that simultaneously had less weight and higher flutter resistance than the baseline model. The best designs of each parametric study are plotted in Figure 11, many of which showed significant improvement (higher flutter speed and/or lower weight) over the baseline. The legend in the figure identifies the studies from which the data points originated. The last entry in the legend is a group of designs that were created from combining designs generated from the separate studies on rib, spar, and stringer modifications. 
Figure 12 illustrates four designs from Figure 11, the first being the baseline CRM model. The Appendix provides data on the weight, stress, and flutter speeds for designs ' $a$ ' through ' $\mathrm{v}$ ' of Figure 11 and are measured as percent changes from the baseline. In Figure 11, two designs which modified the curvature of the spars and stringers (design ' $c$ ' and design ' $\mathrm{f}$ ', where design ' $\mathrm{f}$ ' is the spar configuration in Figure 12(b-d)) showed improvement in the KS value, weight, and flutter speed. The combination of a lower weight, higher flutter resistance, and lower stresses was rarely observed. Of the designs, the largest increase in the KS value (i.e. the stresses) using curvilinear members was $4.4 \%$. For straight-rotated members, the largest increase was $7.3 \%$, but some of this stress may have been due to the configuration of the baseline, where the rib between the inboard and outboard sections remained straight, causing the rotated straight ribs to attach to it. If this rib between the inboard and outboard sections was eliminated, the rotated ribs would remain continuous, potentially decreasing the stresses. Design 'o' in Figure 12 shows the connectivity between the straight-rotated ribs at the boundary between the inboard and outboard ribs.

When using the same set of parameter values to modify the spar/rib curvatures, the spars and stringers proved to be more effective in increasing the flutter point of the designs than the ribs. For example, design ' $\mathrm{f}$ ' (which has spar/stringer modification only) had a $9.3 \%$ improvement in the flutter speed and a $0.5 \%$ decrease in KS, i.e. stress, where design ' $h$ ' (which has rib modification only) had a 3.4\% improvement in flutter speed and a 1.2\% increase in KS. Design 'e' (which had stringer modifications only and no inner spar) had a $6.0 \%$ improvement in the flutter speed and a $2.8 \%$ increase in KS which is expected given the larger weight decrease of 7.1\%. Design ' $\mathrm{f}$ ' and design ' $\mathrm{h}$ ' only had a $1.0 \%$ and $0.2 \%$ decrease in weight, respectively. The spars and stringers may be more effective in increasing the flutter speed than the ribs since they are aligned more with the load path, even when ribs are curved or reoriented. This enables the spars and stringers to have both an inertial and stiffness impact, and the ribs only an inertial impact.

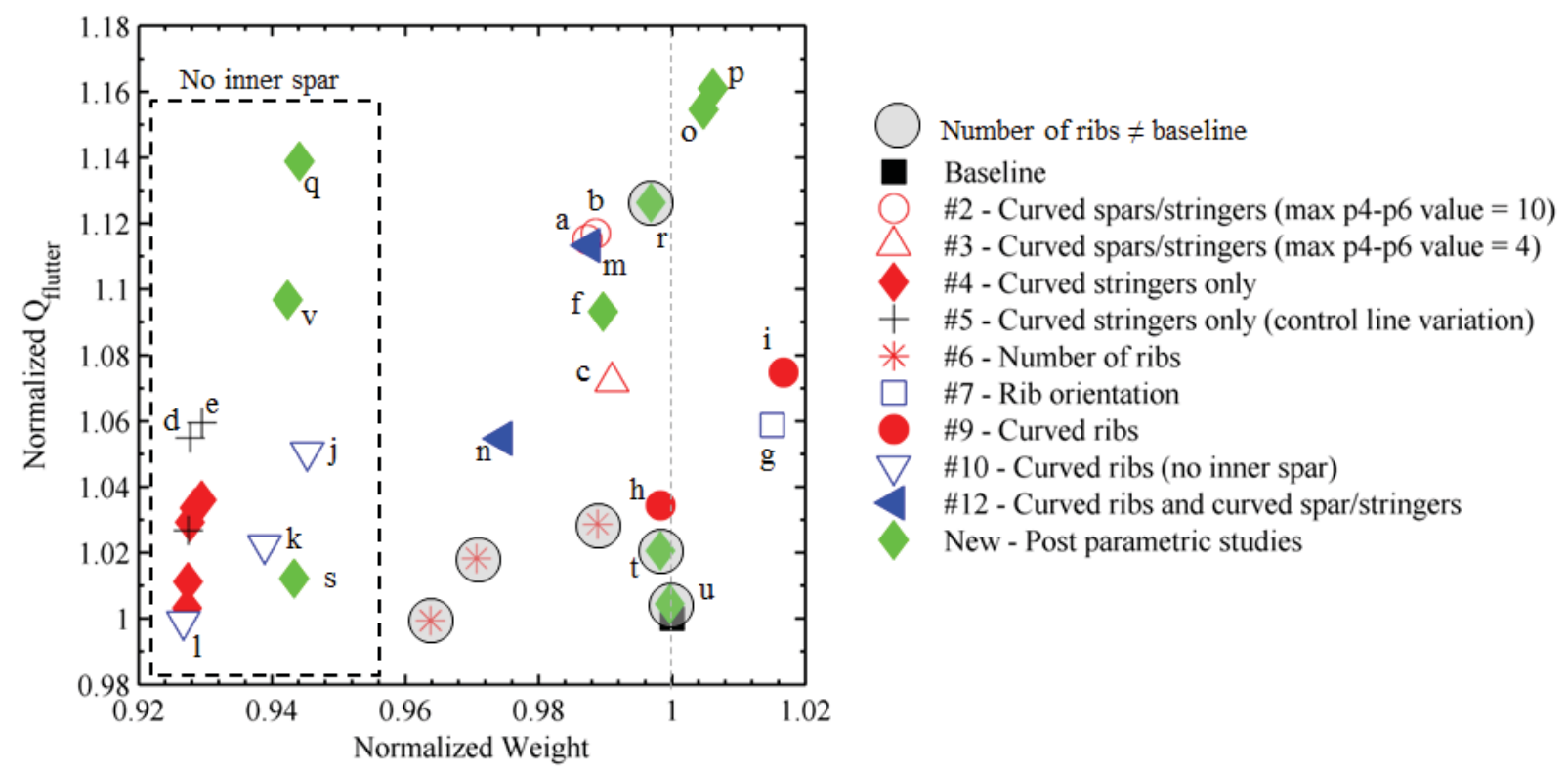

Figure 11. Best designs of the parametric studies along with a nine new designs. The numbers in the legend indicate which of the twelve parametric studies the design came from. The dashed box indicates the designs having no inner spar.

When modifying the spar and stringers (not the ribs), the straight spar/stringer designs (represented as design ' $b$ ' in Figure 11) performed just as well as the curvilinear designs (represented by design ' $a$ '), since the weight and flutter improvements resulted mostly from shifting the spanwise structural members forward. Here, it was most beneficial to straighten the spar and stringers so that a majority of their length was closer to the leading edge. At times curvature did help improve the wing's performance, but this was only when the curvature allowed the spar to bow closer to the leading edge (e.g., design 'c' which has a straight spar in the outboard wing section was modified to have slight curvature, resulting in design ' $\mathrm{f}$ ' which has a higher flutter speed than design ' $c$ '). A straight spar would still outperform this design if its location was closer to the leading edge (e.g., design 'b' has as straight spar 
closer to the leading edge than design ' $\mathrm{f}$ ', which has a slightly curved spar.) When considering buckling, it was found that the straight-spar design had a $10 \%$ reduction in buckling resistance. To maintain the buckling resistance of the baseline yet improve the flutter speed, a particular design used a curvilinear spar and stringer configuration in the inboard section (to improve the support of the skins near the wing root) and a straight, forward-shifted spar and stringers configuration in the outboard (to increase the wing's stability).

When modifying the ribs only (not the spar and stringers), the best designs had a majority (or all) of the rib length oriented at roughly 24-26 degrees (e.g., designs ' $h$ ', 'g', and 'i', whose rib configurations are found in Figure 12(b-d), respectively). Designs ' $g$ ' and ' $i$ ' showed that the straight rib designs performed similarly to the curved rib designs. The straight rib design had more stress but as mentioned above this may have been due to the configuration of the baseline. The curved rib designs had relatively higher weight than the baseline, unless the curved ribs were shifted outboard (design ' $h$ ') due to the values of the rib design parameters or there was a reduction in the number of ribs (design ' $r$ ', 't', and ' $u$ '), which may be feasible since fewer ribs may be sufficient when their relative length is greater. Three designs (' $h$ ', 't', and 'u') had similar weight as the baseline. These designs showed at most a 3.4\% increase in the flutter speed. The third design ('u') had straight-rotated ribs and showed only a $0.4 \%$ improvement in the flutter speed. The second design (' $t$ ') had a lower flutter speed (and ten fewer ribs) than the first (design ' $h$ '), which meant that before the ten ribs were removed, the design's flutter speed (i.e., the flutter speed of design 'i') was influenced by both rib curvature and the additional weight and stiffness from the extra ribs.

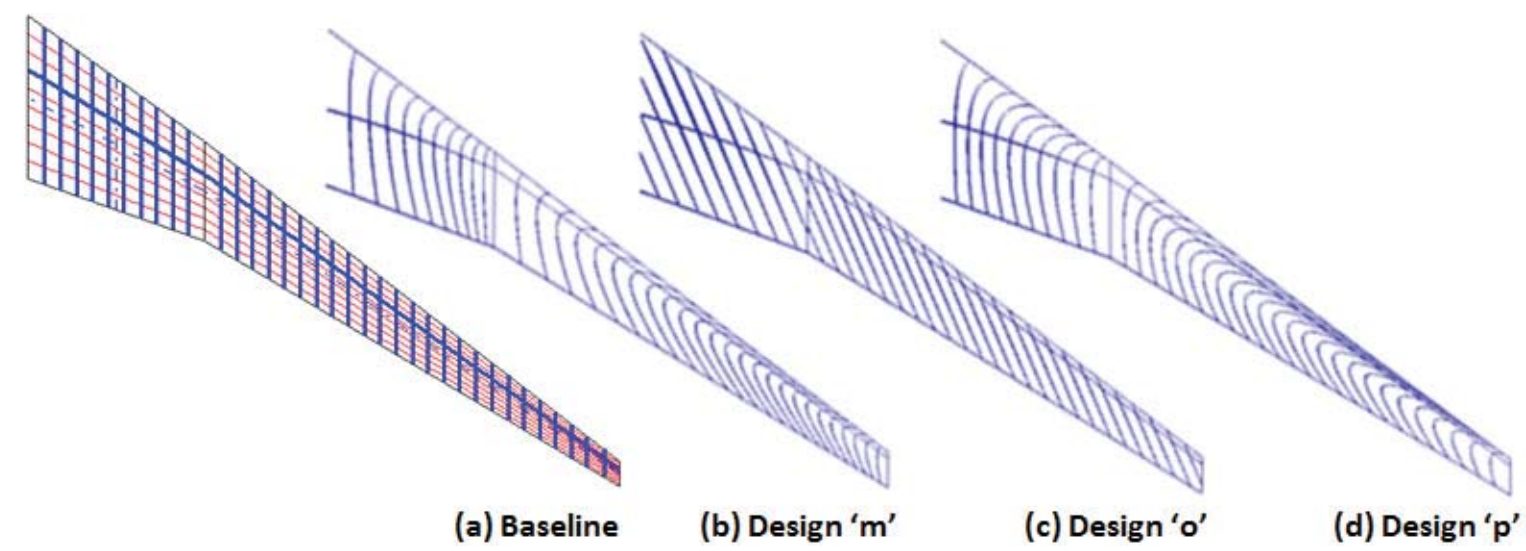

Figure 12. The baseline design and three higher performing designs (where each design has the same number of spars, stringers, and ribs). For designs ' $m$ ', ' $o$ ', and ' $p$ ', the spar configuration from design ' $f$ ' is used along with the rib configurations from designs ' $h$ ', ' $g$ ', and ' $i$ ', respectively Stringers (thin red lines) and parameterization lines (dashed blue lines) are only shown in (a).

Finally, when removing the inner spar from design 'i' (which has curvilinear ribs) and design 'g' (which has rotated straight-ribs), the resulting designs (' $\mathrm{j}$ ' and 's', respectively) showed a decrease in flutter speed, but design ' $j$ ' (having curved ribs) showed less decrease. Designs ' $i$ ' and ' $j$ ' have the greatest rib curvature near the location of the inner spar, suggesting that the rib curvature partially makes up for the lost stiffness and mass of the missing spar where the straight rotated ribs do not have this feature. This was further investigated with a larger group of designs where the results showed that the flutter speed decreased by $2-8 \%$ due to removal of the inner spar. These initial studies suggest that for the same weight, designs having generally more rib curvature tended to have less of a flutter penalty in the absence of the inner spar.

The results of these parametric studies provided additional insight into the following:

- The addition of spars increases the flutter resistance of the wing with a corresponding weight penalty. When spars are located toward the leading edge, the flutter point increases, such that a wing design with 4 spars (and 4 stringers) can have the same flutter resistance as a wing design with 8 spars (and 0 stringers). This follows a well-known trend of pushing the CG forward for better flutter resistance [22].

- The outboard wing section, especially the wing tip, was the most sensitive region for aeroelastic tailoring. For this design space, inertial forces (which are most readily impacted by adding/altering material at the wing tip) are playing a greater role than elastic forces (which are more sensitive to changes at the wing root, where the bending and torsional stresses will be largest). 
- When modifying the number of ribs in a design, there were a few designs with lower weight and higher flutter speed than the baseline, but they required fewer ribs in the inboard and more ribs in the outboard, making the rib spacing quite different between the inboard and outboard wing sections. It is likely this rib configuration increased the wing's resistance to flutter by lowering the frequency of the first bending mode [22]. However, this rib arrangement potentially creates other problems such as buckling or outer mold line distortion.

- When rotating straight ribs, there were clear trends between weight and flutter, with a maximum flutter point at 24 degrees and minimum at -36 degrees; however, no design was superior to the baseline in both weight and flutter. Similar results were found in [18]. The design having ribs oriented at -36 degrees is a standard rib configuration, i.e. ribs are perpendicular to the wing leading edge, which is recommended for weight reduction [23]. This design had $2 \%$ less weight than the baseline, where the design having maximum flutter speed had $1.5 \%$ more weight than the baseline.

- When investigating the effect of rib curvature, the most insight came by comparing designs that had the same curvature definitions (which defined the general curvature as concave or convex) but different control lines (where the control line defined the location of maximum curvature in a rib or spar). Four example cases were considered. Regardless of the direction of the curve (concave or convex), the best designs had a large portion of their curved ribs aligned near 26 degrees. The locations of control lines caused the rib curvatures to align the majority of their rib length at approximately the same orientation as the best design from the rotated, straight-ribs study.

- When combining a high-performing spar design with a high-performing rib design (where the performance is measured with respect to weight and flutter), the resulting designs complimented one another to produce a higher performing design in terms of flutter resistance and weight reduction than the two designs it comprises (neglecting changes in stress).

This last observation of performance superposition of spars and ribs was also explored when analyzing trends among various metrics, including: KS, flexural axis position, tip deflection, tip twist, CG locations, buckling, etc. By exploring obvious trends in the data, no evaluation metric consistently correlated with weight or flutter; however, some trends were detected when comparing designs that had gradual changes in structural configuration from one design to the next, as opposed to picking random designs of varying topology to compare. An expected trend found from the data was that a decrease in weight typically resulted in more tip deflection and higher stresses. It appeared that some wing designs used wash-in to increase flutter resistance (rotated rib designs), which is the expected trend [1], while other designs used wash-out (spar/stringers designs). However when these differing groups of design were merged together, their benefits surprisingly complimented one another (neglecting stress). The data that led to this conclusion is shown in Table 2.

Table 2. Percent differences with respect to the baseline of three designs, where the first two designs combine to make the third design. The rotated ribs design uses baseline spar/stringers, and the curved spar design uses baseline ribs.

\begin{tabular}{lccc|ccc|cc} 
& \multicolumn{9}{c}{ Stiffness } & \multicolumn{2}{c}{ Mass } \\
\hline Design & Weight & KS & $\begin{array}{c}\text { Flutter } \\
\text { point }\end{array}$ & $\begin{array}{c}\text { Flexural } \\
\text { axis* }\end{array}$ & Tip def. & Tip twist ** & $\begin{array}{c}\mathrm{CG}_{\text {root }} \\
(+=\text { aft })\end{array}$ & $\begin{array}{c}\mathrm{CG}_{\text {tip }} \\
(+=\text { aft })\end{array}$ \\
\hline Rotated ribs & 1.5 & 3.8 & 5.8 & 0.2 & 2.7 & -4.8 & -0.12 & 0.01 \\
Curved spar & -1.0 & -0.5 & 9.3 & -0.5 & -0.2 & 2.4 & 0.44 & -0.07 \\
\hline Combination & $\mathbf{0 . 5}$ & $\mathbf{4 . 4}$ & $\mathbf{1 5 . 5}$ & $\mathbf{- 0 . 2}$ & $\mathbf{2 . 6}$ & $\mathbf{- 1 . 9}$ & $\mathbf{0 . 3 3}$ & $\mathbf{- 0 . 0 5}$ \\
\hline
\end{tabular}

* Flexural axis: Positive value indicates flexural axis moving toward wing (more wash-in expected)

** Tip twist: Positive value indicates increased negative twist (more wash-out expected)

The values in Table 2 represent percent differences when compared to the baseline. Looking at the data most influenced by the stiffness (flexural axis, tip deflection, and tip twist), the two designs have clearly differing values as anticipated. Comparing the data most influenced by the mass, the two designs differ once again, since the rib design has its root mass forward of the baseline and its tip mass aft of the baseline. The spar design is just the opposite (noting that movement of the tip mass will have a greater impact on flutter than the root mass). Although these designs seem to have different means to increasing their flutter resistance over the baseline, when combined, their respective increases in flutter point essentially add to create a design with even higher resistance to flutter. In 
the resulting design (provided in the last row of the table and shown as design 'o' in Figure 12), the tip mass moves forward, which has been seen to increase the flutter speed with the spar studies. The flexural axis moves away from the wing, indicating more wash-out than the baseline; however, the wing's twist value is negative, indicating more wash-in behavior than the baseline (at least at the tip). Additionally, when observing the KS values of the two designs, one is positive and one is negative. Typically, for the other metrics in the table (besides flutter), the result for the combined design is somewhere between the two values of the original designs, but here, the resulting KS value for the combined design has a higher absolute value (i.e. higher stress) than either of the other designs it comprises. This example is another demonstration of the difficulties in discovering design trends for improving flutter resistance. Additional details regarding these spar and rib results will be published as a NASA Technical Memorandum.

\section{Summary of Results}

The previous three sections explored aeroelastic tailoring using composite fiber tow steering, thickness variations and functionally graded metals, and shape/topological variations of the spars, ribs, and stringers. The wing skins were the focus of the tow steering studies. The parameterization enabled various composite laminate definitions and within those definitions, allowed for a linear (or piecewise linear) variation of the steering angle from root to tip, resulting in curvilinear steering paths. Additionally, differences in the upper and lower skins were also considered. As the amount of tailorability increased, the KS values correspondingly decreased, indicating that the primary benefit of tow steered composites in skins is to minimize local effects. Of the three tailoring schemes applied to the baseline structure, the lowest static wing stresses were obtained with tow steered skins (47\% improvement), and many of these designs could reduce weight as well (up to 14\%). In contrast, flutter resistance and weight show a strong trade-off for tow steering, where designs having a $14 \%$ less weight had at least a $25 \%$ decrease in flutter resistance, making low-weight, low-stress designs poor-performers for flutter. However, a flutter improvement of $100 \%$ was still observed at a $3.5 \%$ weight reduction for tow steered designs.

The functionally graded material studies focused individually on the skin, spars, and ribs. Each structural component was given a bilinear grading using materials-only, thickness-only, or a combination of material and thickness grading. In the skins, the genetic algorithm used to assess optimal behavior always drove the functionally graded materials to $100 \%$ of the highest stiffness material. Thus, bilinear material grading showed no benefit in the skins, but moderate flutter speed improvements (with no weight or stress increase) could be obtained by grading the spars $(4.8 \%)$ or ribs $(3.2 \%)$, where the best flutter results were obtained by grading both thickness and material. It is possible that material grading is more effective when used locally, such as in high stress regions.

Finally, the curvilinear spar/rib studies focused on the geometric effects of the ribs, stringers, and inner spar. The number, location, and orientation of each structural member were first considered using straight members. Then design parameters that defined the curvature of the spar/stringers and ribs were varied using a full factorial design matrix approach, when applicable, to thoroughly explore the design space. For the topology work, large weight reductions were obtained by removing the inner spar, and performance was maintained by shifting stringers forward and/or using curvilinear ribs: 5.6\% weight reduction, a 13.9\% improvement in flutter speed, but a $3.0 \%$ increase in stress levels. Performance was also maintained (for flutter, not stress) using straight-rotated ribs but the design had a $4.2 \%$ lower flutter speed than the curved ribs of similar weight. Finally, initial studies suggest that when the inner spar is eliminated, the curvature within the ribs potentially makes up for some of the lost stiffness and mass of the inner spar. Allowing the ribs, stringers and spars to curve resulted in greater tailorability of the structural performance, and provided some designs where all three parameters, weight, flutter point, and KS values, were all improved.

Each of the three tailoring studies used the same baseline model, the CRM, as the starting point. Direct comparisons between the different tailoring schemes cannot be made for multiple reasons. First, each tailoring approach has a very large number of design possibilities. To make the studies manageable and insightful, these possibilities are inherently scaled down using parameterization schemes. Since the three tailoring schemes in this report are quite different from each other, it is impossible to ensure an equivalent level of fidelity among their parameterizations. For example, the functionally graded materials study explored the design space using a bilinear grading, which offered a comprehensive approach and a more equivalent comparison to the bilinear thickness-only grading results; however, functionally graded materials may prove to be most beneficial when used more locally, such as in high stress regions. Furthermore, this design space was limited to two materials.

Secondly, even if each tailoring scheme had an equivalent level of fidelity among their parameterizations, locating the optimal design within each scheme would require a more thorough optimization approach. The goal of this work was not design optimization but to learn which tailoring schemes have the most merit before additional 
resources are employed to further exploit these schemes. Additionally, the curvilinear spar/rib studies were less amenable to design optimization since each design permutation's function evaluation required a time intensive remeshing process.

Third, the nature of parametric studies requires that certain parameters or metrics be held constant while the remaining parameters are varied for comparison purposes. Since the focus of this work is weight reduction, it would have been useful to hold the stress value or flutter point constant and monitor the subsequent allowable weight change, but this is very challenging without optimization. Instead it was typically effective to hold the weight constant, as with the functionally graded materials studies, and compare the relative changes in stress and flutter results. With this approach, although the resulting designs all have the same weight, it can be inferred that designs having higher flutter points could be redesigned to a lower weight than the designs with lower flutter points. However, multiple metrics, such as the effect on stress, also need to be taken into account, which tends to complicate these inferences.

\section{Future Work}

These studies provide insight into the individual benefits of tow steering, thickness variations, functionally graded materials, and variations in ribs, stringers and spars. First, formal optimization should be used to further locate the capabilities and trade-offs of the tailoring schemes discussed above. As gradient-based optimization is typically the most cost-effective method (especially for systems with larger numbers of design variables), it will be used for this purpose. Mass will be minimized under constraints on both the KS function and the flutter dynamic pressure such that neither metric can become worse than the baseline structure. The next step is to select the most promising of these technologies applied to the regions to which they have the greatest benefits. For example, exploring a design space in which the skins are tow steered composites, while the internal ribs, spars and stringers are optimized for thickness, functionally graded metallic materials, curvilinearity, placement and spacing.

The tradeoffs between straight and curvilinear members are significant enough that formal design optimization should expose new topological insights. Work is being supported at Virginia Tech to design wings having curvilinear spar/ribs [15] and curvilinear panel stiffeners [14] using gradient and non-gradient based optimization, specifically minimizing their mass under a variety of failure constraints.

Alternative work is underway which considers three-dimensional topology optimization of a wing box [26]: the wing is filled with a large number of three-dimensional finite elements, and the gradient-based optimizer can decide which elements to remove. Material grading and wing skin tailoring, either via tow steered composites or graded materials may be added to this topology optimization process. The optimizer now makes two choices about each element: whether it is solid or void, and its material fraction if it is solid.

Furthermore, it may be beneficial to enable material grading or tow steering to significantly vary within a small section of the wing span similar to the curvilinear panel stiffeners work above, allowing the technologies to have a more local impact. For example, it has been shown that tow steered panels have greater buckling performance than non-steered panels [27]. An opportunity like this may allow for larger rib spacing with fewer ribs within the wing structure.

Beyond the static aeroelastic behavior and flutter behavior, additional load cases and aeroelastic metrics need to be considered in this optimization process. These additional load cases, adding in factors such as engine nacelles and fuel tanks, will influence the KS values and ultimately increase the weight as the structures will be required to sustain all load cases, not just the few selected for the initial technology screening tests. Even with the structural aeroelastic tailoring, the goal of weight reduction is anticipated to increase the flexibility of the wings, particularly as simultaneous requirements within the program drive the wings to higher aspect ratios and less sweep to enable natural laminar flow. Non-conventional control effectors, such as continuous trailing edge effectors, may also be considered to control wing flexibility and exploit it for drag reduction goals elsewhere in the program. This will lead to the use of both passive and active structural concepts. Beyond the inclusion of steady control surface effectiveness as a design constraint, control effectors may eventually be designed to actively suppress flutter instability mechanisms.

\section{Acknowledgements}

This work is funded by the Fixed Wing project under NASA's Fundamental Aeronautics Program. 


\section{References}

[1] Shirk, M., Hertz, T., Weisshaar, T., "Aeroelastic Tailoring - Theory, Practice, Promise," Journal of Aircraft, Vol. 23, No. 1, pp. 6-18, 1986.

[2] Weisshaar, T., "Aircraft Aeroelastic Design and Analysis," 1995 (second edition - 2009) https://engineering.purdue.edu/AAE/Academics/Courses/aae556/2010/Class\%20notes\%20Chapter\%201., Feb, 21, 2012.

[3] Vassberg, J., DeHaan, M., Rivers, S., Wahls, R., "Development of a Common Research Model for Applied CFD Studies." AIAA Applied Aerodynamics Conference, Honolulu, HI, August, 2008.

[4] Taminger, K., Hafley, R., "Electron Beam Freeform Fabrication: A Rapid Metal Deposition Process." Proceedings of the 3rd Annual Automotive Composites Conference, Troy, MI, September 9-10, 2003.

[5] Ijsselmuiden, S., Abdalla, M., Gürdal, Z., "Optimization of Variable-Stiffness Panels for Maximum Buckling Load Using Lamination Parameters," AIAA Journal, Vol. 48, No. 1, pp. 134-143, 2010.

[6] Honda, S., Narita, Y., Sasaki, K., "Maximizing the Fundamental Frequency of Laminated Composite Plates With Optimally Shaped Curvilinear Fibers," Journal of System Design and Dynamics, Vol. 3, No. 6, pp. 867876, 2009.

[7] Lopes, C., Gürdal, Z., Camanho, P., "Tailoring for Strength of Composite Steered-Fibre Panels with Cutouts," Composites Part A: Applied Science and Manufacturing, Vol. 41, No. 12, pp. 1760-1767, 2010.

[8] Haddadpour, H., Zamani, Z., "Curvilinear Fiber Optimization Tools for Aeroelastic Design of Composite Wings," Journal of Fluids and Structures, Vol. 33, pp. 180-190, 2012.

[9] Stodieck, O., Cooper, J., Weaver, P., Kealy, P., "Improved Aeroelastic Tailoring Using Tow-Steered Composites," Composite Structures, Vol. 106, pp. 703-715, 2013.

[10] Marzocca, P., Fazelzadeh, S., Hosseini, M., "A Review of Nonlinear Aero-Thermo-Elasticity of Functionally Graded Panels," Journal of Thermal Stresses, Vol. 34, pp. 536-568, 2011.

[11] Birman, V., Byrd, L., "Modeling and Analysis of Functionally Graded Materials and Structures," Applied Mechanics Reviews, Vol. 60, No. 5, pp. 195-216, 2007.

[12] Librescu, L., Maalawi, K., "Material Grading for Improved Aeroelastic Stability in Composite Wings," Journal of Mechanics of Materials and Structures, Vol. 2, No. 7, pp. 1381-1394, 2007.

[13] Dang, T., Kapania, R., Slemp, W., Bhatia, M., Gurav, S., "Optimization and Postbuckling Analysis of Curvilinear-Stiffened Panels Under Multiple-Load Cases," Journal of Aircraft, Vol. 47, No. 5, pp. 1-12, 2010.

[14] Bhatia, M., Kapania, R., Evans, D., "Comparative Study on Optimal Stiffener Placement for Curvilinearly Stiffened Panels," Journal of Aircraft, Vol. 48, No. 1, pp. 77-91, 2011.

[15] Locatelli, D., Mulani, S., Kapania, R., "Wing-Box Weight Optimization Using Curvilinear Spars and Ribs (SpaRibs)," Journal of Aircraft, Vol. 48, No. 5, pp. 68-79, 2011.

[16] Balabanov, V., Haftka, R., "Topology Optimization of a Transport Wing Internal Structure,” AIAA Paper 944414.

[17] Kobayashi, M., Pedro, H., Kolonay, R., Reich, G., "On a Cellular Division Method for Aircraft Structural Design,” The Aeronautical Journal, Vol. 113, No. 1150, pp. 821-831, 2009.

[18] Harmin, M., Ahmed, A., Cooper, J., Bron, F., "Aeroelastic Tailoring of Metallic Wing Structures," AIAA Structures, Structural Dynamics and Materials Conference, Denver, Colorado, April 4-7, 2011.

[19] Kreisselmeier, G., Steinhauser, R., "Systematic Control Design by Optimizing a Vector Performance Index", International Federation of Active Controls Symposium on Computer-Aided Design of Control Systems, Zurich, Switzerland, 1979.

[20] Weisshaar, T., Nam, C., Batista-Rodriguez, A., "Aeroelastic Tailoring for Improved UAV Performance," AIAA Structures, Structural Dynamics, and Materials Conference, Long Beach, CA, April 20-23, 1998.

[21] Deb, K., Pratap, A., Agarwal, S., Meyarivan, T., "A Fast and Elitist Multiobjective Genetic Algorithm," IEEE Transactions on Evolutionary Computations, Vol. 6, No. 2, pp. 182-197, 2002.

[22] Bisplinghoff, R., Ashley, H., Halfman, R., Aeroelasticity, Dover Publications, Mineola, NY, 1996.

[23] Niu, M., Airframe Structural Design, Hong Kong Conmilit Press, Hong Kong, 1988.

[24] Locatelli, D., "Optimization of Supersonic Aircraft Wing-Box Using Curvilinear SpaRibs," PhD Dissertation, Virginia Polytechnic Institute and State University, 2012.

[25] Box, G.E., Hunter, J.S., Hunter,W.G. Statistics for Experimenters: Design, Innovation, and Discovery, 2nd Edition, Wiley, Hoboken, New Jersey, 2005.

[26] Dunning, P. D., Brampton, C. J. and Kim, H. A., "Multidisciplinary Level Set Topology Optimization of the Internal Structure of an Aircraft Wing," 10th World Congress on Structural and Multidisciplinary Optimization, Orlando, Florida, USA, May 19-24, 2013. 
[27] Weaver, P.M., Potter, K.D., Hazra, K., Saverymuthapulle, M.A.R., Hawthorne, M.T., "Buckling of variable angle tow plates: from concept to experiment." 50th AIAA/ASME/ASCE/AHS/ASC Structures, Structural Dynamics, and Materials Conference, Palm Springs, California, May 4-7, 2009.

\section{Appendix}

Table 3. Percent changes of the curvilinear spar/ribs designs with respect to the baseline model. (Positive and negative values are distinguished by color.)

\begin{tabular}{|c|c|c|c|}
\hline $\begin{array}{c}\text { weight } \\
(\%)\end{array}$ & $\begin{array}{l}\mathrm{KS} \\
(\%)\end{array}$ & $\begin{array}{c}\text { flutter } \\
\text { speed } \\
(\%)\end{array}$ & Design description \\
\hline-1.27 & 1.09 & 11.52 & $\begin{array}{l}\text { Design a, inner spar and stringers curved in IBD, not curved but shifted } \\
\text { forward in OBD, ribs = baseline }\end{array}$ \\
\hline-1.15 & 1.10 & 11.70 & $\begin{array}{l}\text { Design b, inner spar and stringers not curved but shifted forward across entire } \\
\text { span, ribs = baseline }\end{array}$ \\
\hline-0.90 & -0.22 & 7.21 & Design c, like design a but not shifted forward as much, ribs = baseline \\
\hline-7.23 & 2.41 & 5.50 & $\begin{array}{l}\text { Design d, no inner spar, stringers like design c but with OBD spar bowed } \\
\text { forward, ribs = baseline }\end{array}$ \\
\hline-7.05 & 2.83 & 5.95 & Design e, similar to design d, ribs = baseline \\
\hline-1.03 & -0.49 & 9.32 & Design $\mathrm{f}$, similar to design d but with an inner spar, ribs $=$ baseline \\
\hline 1.51 & 3.76 & 5.79 & Design $\mathrm{g}$, straight ribs rotated at 24 degrees, spar/stringers = baseline \\
\hline-0.17 & 1.25 & 3.44 & Design $\mathrm{h}$, curved rib design (weight $<$ baseline), spar/stringers $=$ baseline \\
\hline 1.68 & -0.07 & 7.47 & Design i, curved rib design (weight $>$ baseline), spar/stringers = baseline \\
\hline-5.47 & 2.90 & 5.05 & Design $\mathrm{j}$, no inner spar, same rib configuration as design $\mathrm{i}$, stringers $=$ baseline \\
\hline-6.11 & 3.29 & 2.23 & Design $\mathrm{k}$, slightly different curvature than design $\mathrm{j}$ \\
\hline-7.33 & 4.44 & -0.07 & Design 1 , no inner spar, same rib configuration as design $\mathrm{h}$, stringers $=$ baseline \\
\hline-1.23 & 1.26 & 11.33 & Design $\mathrm{m}$, spar/stringers $=$ design $\mathrm{f}$, ribs $=$ design $\mathrm{h}$ \\
\hline-2.55 & 0.45 & 5.47 & $\begin{array}{l}\text { Design } \mathrm{n} \text {, spar/stringers = design } \mathrm{f} \text {, trivial design due to ribs being straight and } \\
\text { shifted outboard }\end{array}$ \\
\hline 0.48 & 4.44 & 15.45 & Design $\mathrm{o}$, spar/stringers $=$ design $\mathrm{f}$, ribs $=$ design $\mathrm{g}$ \\
\hline 0.61 & 0.41 & 16.10 & Design $\mathrm{p}$, spar/stringers $=$ design $\mathrm{f}$, ribs $=$ design $\mathrm{i}$ \\
\hline-5.59 & 2.95 & 13.89 & Design $\mathrm{q}$, stringers $=$ design $\mathrm{d}$, ribs $=$ design $\mathrm{i}$ \\
\hline-0.31 & 0.64 & 12.63 & Design $\mathrm{r}$, spar/stringers $=$ design $\mathrm{f}$, ribs $=\operatorname{design} \mathrm{i}(5$ less OBD ribs $)$ \\
\hline-5.67 & 7.06 & 1.21 & Design $\mathrm{s}$, no inner spar, stringers $=$ baseline, ribs $=$ design $\mathrm{g}$ \\
\hline-0.17 & 0.32 & 2.06 & Design $\mathrm{t}$, spar/stringers $=$ baseline, ribs $=$ design $\mathrm{i}(10$ less OBD ribs $)$ \\
\hline-0.03 & 1.65 & 0.45 & Design $\mathrm{u}$, spar/stringers $=$ baseline, ribs $=$ design $\mathrm{g}(9$ less OBD ribs $)$ \\
\hline-5.77 & 7.27 & 9.68 & Design $\mathrm{v}$, no inner spar, stringers $=$ design $\mathrm{d}$, ribs $=$ design $\mathrm{g}$ \\
\hline
\end{tabular}

